\title{
HARMONIC DIFFEOMORPHISMS INTO CARTAN-HADAMARD SURFACES WITH PRESCRIBED HOPF DIFFERENTIALS
}

\author{
LUEN-FAI TAM AND TOM Y. H. WAN
}

\section{INTRODUCTION}

In [22], the second author showed that given a holomorphic quadratic differential on the unit disk of the complex plane $\mathbb{C}$, one can construct a harmonic diffeomorphism from the hyperbolic two space $\mathbb{H}$ into itself so that its Hopf differential is equal to the given one. Later, in [4], $\mathrm{Au}$ and the second author generalized the result for holomorphic quadratic differentials on $\mathbb{C}$, provided the differential is not of the form $c d z^{2}$ for some constant $c$. Even though it is not explicitly stated in $[22,4]$, the harmonic diffeomorphism is unique up to an isometry in the target. The method of proof in $[22,4]$ is to construct a complete constant mean curvature space-like surface (a constant mean curvature cut) in the Minkowski three space from the given holomorphic quadratic differential. The required harmonic diffeomorphism is just the Gauss map of the constant mean curvature cut.

On the other hand, Li, Wang and the first author [17] studied a class of surfaces which is more general than the hyperbolic two space. They studied complete simply connected surfaces with Gaussian curvatures bounded between a negative constant and 0 , so that the first eigenvalue for the Laplacian for functions is positive. Following [17], we call such a surface a hyperbolic Cartan-Hadamard surface, or simply a hyperbolic $\mathrm{CH}$ surface. For example, a simply connected surface with Gaussian curvature pinched between two negative constants is a hyperbolic $\mathrm{CH}$ surface. It was proved in [17] that a

The first author is partially supported by NSF Grant \# DMS 9300422 .

The second author is partially supported by the Earmarked Grant, Hong Kong. 
hyperbolic $\mathrm{CH}$ surface is conformally equivalent to the hyperbolic surface with a conformal factor bounded between two positive constants. It was proved in [17], many things that are true for harmonic maps between hyperbolic two spaces, are still true for harmonic maps between hyperbolic $\mathrm{CH}$ surfaces. For example, in [17], the results in $[14,15,16]$ on the existence, uniqueness and regularity on harmonic maps between hyperbolic spaces with prescribed boundary data at the geometric boundary are still true for hyperbolic $\mathrm{CH}$ surfaces. It was proved in [22] that a harmonic diffeomorphism between hyperbolic surface is quasi-conformal if and only if the norm of its Hopf differential is uniformly bounded. This has also been generalized to hyperbolic $\mathrm{CH}$ surfaces in [17].

It is interesting to see whether one can generalize the results in $[22,4]$ on the constructions of harmonic diffeomorphisms with prescribed Hopf differential. More precisely, given a holomorphic quadratic differential on the unit disk in $\mathbb{C}$ or on the whole complex plane $\mathbb{C}$, one would like to construct a harmonic map, unique up to isometries of the target, from the hyperbolic space or $\mathbb{C}$ into a hyperbolic $\mathrm{CH}$ surface, so that its Hopf differential is equal to the given holomorphic quadratic differential. In this situation, the target is not the hyperbolic space, and one cannot use the Gauss maps of constant mean curvature cuts as in $[22,4]$. New methods have to be developed. In this paper, we are able to solve the existence problem completely and obtain the following:

Main Theorem (Theorem 3.2). Let $N=\left(\mathbb{D}, e^{2 \psi} d s_{p}^{2}\right)$ be a hyperbolic $C H$ surface with Gaussian curvature $K_{N}$ satisfying $-b^{2} \leq K_{N} \leq 0$ for some constant $b>0$ and $\lambda_{1}(N)>0$. Then given any holomorphic quadratic differential $\Phi=\phi d z^{2}$ on $\mathbb{D}_{R_{0}}, R_{0}=1$ or $\infty$, there is a harmonic map $u$ from $\mathbb{D}_{R_{0}}$ to $N$ with Hopf differential given by $\Phi$. Moreover, if $R_{0}=1$ or $\phi$ is not a constant, then $u$ can be chosen to be a harmonic diffeomorphism into $N$. Futhermore, if $R_{0}=1$ and $\Phi \in B D Q(\mathbb{D})$, then $u$ can be chosen to be a quasi-conformal harmonic diffeomorphism onto $N$.

Here, $\mathbb{D}_{1}$ is the unit disk and $\mathbb{D}_{\infty}$ is the complex plane. Since a hyperbolic $\mathrm{CH}$ surface is conformally equivalent to the hyperbolic space with conformal factor bounded between two positive constants as mentioned above, and since a map is harmonic depends only on the conformal structure of the domain 
and not the metric of the domain in dimension two, the theorem is still true if the domain is a hyperbolic $\mathrm{CH}$ surface. In order to prove the theorem without using the properties of constant mean curvature cuts, we have to obtained more refined apriori estimates for the energy density and the dilatation of a harmonic map. One difficulty is that the Gaussian curvature of the target may be zero at some point. Therefore, one cannot use directly the generalized maximum principle as in [22] to obtain a bound of the energy density of the harmonic map in terms of its quadratic differential. However, if the target is a hyperbolic $\mathrm{CH}$ surface, then we can perturb the metric, and use the results in [17] to prove the theorem.

For the uniqueness, we have some partial results. First, we will reduce the question on the uniqueness of harmonic diffeomorphisms to the question on the uniqueness of solutions of the equation satisfied by the $\partial$-energy density of a harmonic map. Then, we will prove, or example:

Theorem 4.5. Let $\mathbb{H}=\left(\mathbb{D}, d s_{p}^{2}\right)$ be the Poincaré disk and let $N$ be a hyperbolic CH surface with Gaussian curvature $K_{N}$. Let $\phi d z^{2}$ be a holomorphic quadratic differential in $B Q D(\mathbb{D})$. Let $u_{1}$ and $u_{2}$ be two orientation preserving harmonic diffeomorphisms from $\mathbb{H}$ into $N$ with the same Hopf differential $\phi d z^{2}$. Suppose $e^{2 w_{i}} d s_{p}^{2}$ is complete on $\mathbb{D}$, for $i=1,2$, where $w_{i}=\log \left\|\partial u_{i}\right\|$, and suppose $K_{N}\left(u_{1}(z)\right)=K_{N}\left(u_{2}(z)\right)$ for all $z \in \mathbb{D}$. Then there is an isometry $\iota_{N}$ of $N$ such that $u_{2}=u_{1} \circ \iota_{N}$.

Here again, we cannot use the properties on Gauss maps of constant mean curvature cuts. One of the main difficulties to prove uniqueness is that the Gaussian curvature of the target may not be constant. However, even if we assume $K_{N}\left(u_{1}\right)=K_{N}\left(u_{2}\right)$ as above, it is still not obvious that $w_{1}=w_{2}$, because $K_{N}$ may equal to 0 somewhere.

There are many other methods and results on constructing harmonic maps on noncompact manifolds, see $[2,3,6,7,9,14,15,16,17,23]$. On the other hand, there are applications of harmonic maps to the Teichmüller theory via Hopf differentials of harmonic maps, see [24, 11]. One of the basic result in this direction is the construction of a homeomorphism between the Teichmüller space of a compact oriented surface of genus great than one and the space of 
holomorphic quadratic differentials on the same surface endowed with a fixed conformal structure in [24] (see also [11]). Later, in [22], by solving the prescribed Hopf differential problem for harmonic diffeomorphisms between $\mathbb{H}$ the second author constructed a continuous mapping from the space of bounded holomorphic quadratic differentials with respect to the Poincaré metric on $\mathbb{H}$ into the universal Teichmüller space which is an extension of the inverse mapping of the homeomorphism constructed in [24]. It was asked by the second author and conjectured by Schoen [19] that this mapping is bijective. The injectivity part of Schoen's conjecture was then proved by $\mathrm{Li}$ and the first author [16], and the surjectivity part is still open. Due to the success of the application of the restricted map on Teichmüller spaces of compact surfaces, this mapping is expected to be very useful in the study of general Teichmüller spaces of Fushian groups especially those of noncompact surfaces. Hence, the generalization of the prescribed Hopf differential problem for harmonic maps in this paper may give more information in the study of the conjecture of Schoen and also in the study of noncompact Riemann surfaces.

Here is our plan. In $\S 1$, we will improve some of the results in [22] for the solution of the nonlinear scalar equation satisfied by the $\partial$-energy density of a harmonic map. In $\S 2$, we will prove our main theorem in the particular case that the domain is $\mathbb{H}$, the norm of the holomorphic quadratic differential is uniformly bounded, and the Gaussian curvature of the target is pinched between two negative constants. In $\S 3$, we will use compact exhaustion and perturbation method to prove the main theorem in its full generality. In $\S 4$, we will prove various results on uniqueness for harmonic maps with the same Hopf differential. In the Appendix, we collect some facts about quasiconformal maps used in this paper for the convenience of the readers. Every surface considered in this paper is assumed to be connected and oriented.

The authors would like to thank Thomas K. K. Au, S.Y. Cheng, and P. $\mathrm{Li}$ for valuable discussions, and J.-P. Wang for his interest in this work. The research is done while the first author is visiting the Chinese University of Hong Kong. He would like to express his gratitude for the hospitality. 


\section{The Equation of $\partial$-Energy Density}

In this paper, $\mathbb{D}_{z, r}$ will denote the disc centered at $z$ of radius $0<r \leq \infty$ in $\mathbb{R}^{2}$. Here, by convention $\mathbb{D}_{z, \infty}$ is just $\mathbb{R}^{2}$. For $0<r<\infty$, the Poincaré metric on $\mathbb{D}_{z, r}$ is denoted by $\mathrm{d} s_{p_{z, r}}^{2}=\rho_{z, r}^{2}(\zeta)|\mathrm{d} \zeta|^{2}$, where $\rho_{z, r}^{2}(\zeta)=4 r^{2} /\left(r^{2}-|z-\zeta|^{2}\right)^{2}$. For simplicity, if $z=0$, then we will denote $\mathbb{D}_{0, r}, \rho_{0, r}$, and $\mathrm{d} s_{p_{0, r}}^{2}$ by $\mathbb{D}_{r}, \rho_{r}$, and $\mathrm{d} s_{p_{r}}^{2}$ respectively. If we also have $r=1$, we simplify the notations further to write $\mathbb{D}, \rho$, and $\mathrm{d} s_{p}^{2}$ instead of $\mathbb{D}_{1}, \rho_{1}$, and $\mathrm{d} s_{p_{1}}^{2}$, respectively.

Let $Q D\left(\mathbb{D}_{r}\right), 0<r<\infty$, be the vector space of holomorphic quadratic differentials on $\mathbb{D}_{r}$ with respect to the standard conformal structure. For $\Phi=\phi(z) \mathrm{d} z^{2} \in Q D\left(\mathbb{D}_{r}\right)$, we set $\|\Phi\|_{\rho_{r}}(z)=\rho_{r}^{-2}(z)|\phi(z)|$ and $\|\Phi\|_{Q D\left(\mathbb{D}_{r}\right)}=$ $\sup _{\mathbb{D}_{r}}\|\Phi\|_{\rho_{r}}(z)$. It is easy to see that $B Q D\left(\mathbb{D}_{r}\right)=B Q D\left(\mathbb{D}_{r}, \mathrm{~d} s_{p_{r}}^{2}\right)=\{\Phi \in$ $\left.Q D\left(\mathbb{D}_{r}\right):\|\Phi\|_{Q D\left(\mathbb{D}_{r}\right)}<\infty\right\}$ is a Banach space with the norm $\|\cdot\|_{Q D\left(\mathbb{D}_{r}\right)}$. To simplify notations, we write $B Q D,\|\Phi\|(z),\|\Phi\|_{Q D}$ to denote the corresponding objects for $r=1$. Since we have global isothermal coordinate $z$ on $\mathbb{D}, \Phi$ is represented uniquely by its coefficient $\phi(z)$. So when there is no confusion, we will not distinguish $\Phi$ and its coefficient $\phi$. For example, $\|\phi\|(z)$ is the same as $\|\Phi\|(z)$.

Let us recall some basic facts about harmonic maps between surfaces. Let $M$ and $N$ be oriented surfaces with metrics $\rho^{2}|\mathrm{~d} z|^{2}$ and $\sigma^{2}|\mathrm{~d} u|^{2}$, where $z$ and $u$ are local complex coordinates of $M$ and $N$ respectively. A $C^{2}$ map $u$ from $M$ to $N$ is harmonic if and only if $u$ satisfies

$$
\frac{\partial^{2} u}{\partial z \partial \bar{z}}+2 \frac{\partial \log \sigma(u(z))}{\partial u} \frac{\partial u}{\partial z} \frac{\partial u}{\partial \bar{z}}=0
$$

The Hopf differential $\Phi=\phi(z) \mathrm{d} z^{2}$ of $u$ is defined by $\phi(z)=\sigma^{2}(u(z)) u_{z}(z) \bar{u}_{z}(z)$. If $u$ is harmonic, then it is well-known that $\Phi$ is a holomorphic quadratic differential on $M$. Let

$$
\|\partial u\|^{2}(z)=\frac{\sigma^{2}(u(z))}{\rho^{2}(z)}\left|\frac{\partial u}{\partial z}\right|^{2}(z),
$$

and

$$
\|\bar{\partial} u\|^{2}(z)=\frac{\sigma^{2}(u(z))}{\rho^{2}(z)}\left|\frac{\partial u}{\partial \bar{z}}\right|^{2}(z),
$$


be the $\partial$ and $\bar{\partial}$-energy densities of $u$ respectively. The energy density and the Jacobian of $u$ are given by

$$
e(u)=\|\partial u\|^{2}+\|\bar{\partial} u\|^{2}
$$

and

$$
J(u)=\|\partial u\|^{2}-\|\bar{\partial} u\|^{2}
$$

respectively. If $u$ is harmonic and $\|\partial u\| \neq 0$, by [20], we have the following Bochner formula:

$$
\triangle_{M} w=-K_{N}(u)\left(e^{2 w}-\|\phi\|_{M}^{2} e^{-2 w}\right)+K_{M},
$$

where $w=\log \|\partial u\|, K_{M}$ and $K_{N}$ are the Gaussian curvatures of $M$ and $N, \Delta_{M}$ is the Laplacian operator on $M$, and $\|\phi\|_{M}$ is the norm of the Hopf differential of $u$ with respect to the metric on $M$. In our case, $M$ is either $\mathbb{H}$ or $\mathbb{R}^{2}$. In this section, we will study the equation

$$
\triangle_{p} w=h(z)\left(e^{2 w}-\|\phi\|_{\rho_{r}}^{2} e^{-2 w}\right)-1
$$

on a disc $\mathbb{D}_{r}, 0<r<\infty$, for some function $h(z)$, where $\triangle_{p}$ is the Laplacian operator with respect to the Poincaré metric $\rho_{r}^{2}(z)|\mathrm{d} z|^{2}$ on $\mathbb{D}_{r}$. The results will be used in the later sections. The analysis is the same for all $r$ with $0<r<\infty$. Hence we will assume that $r=1$ for simplicity.

Let us first recall the following generalized maximum principle of Cheng and Yau [5].

Lemma 1.1. Let $M$ be a complete noncompact manifold with Ricci curvature bounded below by $-K$, for some constant $K \geq 0$. Let $u$ be a $C^{2}$ function on $M$.

(1) Suppose $u$ satisfies the differential inequality $\Delta u \geq f(u)$, where $f$ is a continuous function with the property that

$$
\limsup _{t \rightarrow \infty} \frac{f(t)}{g(t)}>0
$$

for some positive continuous function $g$, which is nondecreasing on $[a, \infty)$ for some $a$ and $\int_{b}^{\infty}\left(\int_{a}^{t} g(\tau) d \tau\right)^{-1 / 2}<\infty$ for some $b>a$. Then $u$ is bounded from above. 
(2) Suppose $u$ is bounded from above, then there is a sequence of points $x_{k}$ in $M$, such that

$$
\begin{gathered}
\lim _{k \rightarrow \infty} u\left(x_{k}\right)=\sup _{M} u, \\
\lim _{k \rightarrow \infty}\left|\nabla u\left(x_{k}\right)\right|=0,
\end{gathered}
$$

and

$$
\limsup _{k \rightarrow \infty} \Delta u\left(x_{k}\right) \leq 0 .
$$

In our situations, we usually take $g(u)=f(u)=A e^{\alpha u}-B e^{-\beta u}-C$ for some constants $\alpha, \beta, A, B$, and $C>0$ to apply Lemma 1.1. Using the generalized maximum principle, we have:

Lemma 1.2. Given $\phi d z^{2} \in Q D\left(\mathbb{D}_{r}\right), r=1$ or $\infty$. Let $w_{1}$ and $w_{2}$ be two $C^{2}$ functions satisfying $|\phi| e^{-2 w_{i}} \leq 1, i=1,2$, such that

$$
\triangle_{0} w_{1} \geq h_{1}(z)\left(e^{2 w_{1}}-|\phi|^{2} e^{-2 w_{1}}\right)
$$

and

$$
\triangle_{0} w_{2} \leq h_{2}(z)\left(e^{2 w_{2}}-|\phi|^{2} e^{-2 w_{2}}\right),
$$

for some continuous nonnegative functions $h_{1}$ and $h_{2}$, where $\Delta_{0}$ is the Euclidean Laplacian. Suppose $e^{2 w_{2}}|d z|^{2}$ defines a complete metric on $\mathbb{D}_{r}, h_{1}(z) \geq$ $h_{2}(z)$, and $b^{2} \geq h_{1}(z) \geq a^{2}>0, \forall z \in \mathbb{D}_{r}$, for some constants $b \geq a>0$, then

$$
w_{1} \leq w_{2} .
$$

Proof. First of all, $|\phi| e^{-2 w_{i}} \leq 1$ implies that $e^{2 w_{i}}-|\phi|^{2} e^{-2 w_{i}} \geq 0, i=1,2$. Hence $h_{1}(z) \geq h_{2}(z) \geq 0$ implies

$$
\begin{aligned}
\triangle_{0} w_{2} & \leq h_{2}(z)\left(e^{2 w_{2}}-|\phi|^{2} e^{-2 w_{2}}\right) \\
& \leq h_{1}(z)\left(e^{2 w_{2}}-|\phi|^{2} e^{-2 w_{2}}\right) .
\end{aligned}
$$

Therefore, letting $\eta=w_{1}-w_{2}$, we get

$$
\begin{aligned}
e^{-2 w_{2}} \triangle_{0} \eta & \geq h_{1}(z)\left(e^{2 \eta}-\left|\mu_{2}\right|^{2} e^{-2 \eta}-1+\left|\mu_{2}\right|^{2}\right) \\
& \geq a^{2} e^{2 \eta}-b^{2} e^{-2 \eta}-b^{2},
\end{aligned}
$$

where $\left|\mu_{2}\right|=|\phi| e^{-2 w_{2}} \leq 1$, and we have used the fact that $b^{2} \geq h_{1}(z) \geq$ $a^{2}>0$. It is easy to see that the curvature of $e^{2 w_{2}}|d z|^{2}$ is bounded below by 
$-h_{2}(z)\left(1-\left|\mu_{2}\right|^{2}\right) \geq-b^{2}$. Since $e^{2 w_{2}}|d z|^{2}$ is complete, Lemma 1.1 implies that $\eta$ is bounded from above. Then, as in [22], the second part of Lemma 1.1 implies that $\eta \leq 0$, i.e. $w_{1} \leq w_{2}$.

Using the corresponding results in [22] for $\mathbb{D}$ and [4] for $\mathbb{R}^{2}$ with $c=1$, we have the following lemma.

Lemma 1.3. For any positive constant $c^{2}>0$, and any $\phi d z^{2} \in Q D\left(\mathbb{D}_{r}\right)$, $r=1$ or $\infty$, with $\phi \not \equiv 0$ if $r=\infty$. There is a unique solution $v$ of the equation

$$
\Delta_{0} v=c^{2}\left(e^{2 v}-|\phi|^{2} e^{-2 v}\right)
$$

on $\mathbb{D}_{r}$, such that $e^{2 v}|d z|^{2}$ is complete on $D_{r}$, and $e^{-2 v}|\phi| \leq 1$ everywhere. Here, $\Delta_{0}$ is the Euclidean Laplacian of $\mathbb{D}_{r}$. Furthermore,

(1) if $r=1$, then $v-\log \rho \geq-\log c$, where $\rho^{2}|d z|^{2}$ is the Poincaré metric on $\mathbb{D}$. If, in addition, $\phi d z^{2} \in B Q D(\mathbb{D})$, then

$$
v-\log \rho \leq \frac{1}{2} \log \left(\frac{1+\sqrt{1+4 c^{4}\|\phi\|_{Q D}^{2}}}{2 c^{2}}\right),
$$

and there exists a constant $0<k<1$, depending only on $c^{2}\|\phi\|_{Q D}$, such that $e^{-2 v}|\phi| \leq k$.

(2) If $r=1$, then $e^{-2 v}|\phi|<1$. If $r=\infty$, then either $e^{-2 v}|\phi|<1$ everywhere, or $v$ is a constant and $\phi$ is a nonzero constant.

Remark 1.1. It is easy to see that there is no solution to the equation $\Delta_{0} v=$ $c^{2} e^{2 v}$ in $\mathbb{R}^{2}$.

Proof of the lemma. For $r=1$, the lemma follows from applying the results in [22] to $v-\log \rho+\log c$ with $\phi$ replaced by $c^{2} \phi$. For $r=\infty$, the existence and uniqueness of $v$ follow from the results in [4]. Therefore, it remains to prove statement (2) of the lemma. Consider the metric $g=\mathrm{d} s^{2}=e^{2 v}|\mathrm{~d} z|^{2}$ which is complete on $\mathbb{R}^{2}$ by construction. Let $K_{g}$ be the Gaussian curvature of $g$. Using the equation of $v$, it is easy to see that $K_{g}=-c^{2}\left(1-|\phi|^{2} e^{-4 v}\right)$. Therefore $-c^{2} \leq K_{g} \leq 0$. Moreover, using the assumption that $e^{-2 v}|\phi| \leq 1$, we have

$$
\Delta_{g}\left(-K_{g}\right) \leq 4\left(-K_{g}\right)
$$


Since $-K_{g} \geq 0$, we conclude, by mean-value inequality, see for example [10], that the set $K_{g}=0$ is open. It is obvious that the set $K_{g}=0$ is close, therefore we have either $K_{g}<0$ everywhere or $K_{g} \equiv 0$. If $K_{g}<0$ everywhere, then we have $e^{-2 v}|\phi|<1$ everywhere. Suppose $K_{g} \equiv 0$, then $\left(\mathbb{D}_{r}, e^{2 v}|d z|^{2}\right)$ is flat, so $r=\infty$, and using a trick in [19] and the result in [13], we can prove that $v$ is a constant. In fact, let $o$ be the origin in $\mathbb{R}^{2}$, and let $r_{o}(x)$ be the Euclidean distance of a point $x$ from $o$. There exists $R>1$ such that $r_{o}(x) \geq 1$ for all $x$ with $d_{g}(o, x) \geq R$, where $d_{g}$ is the distance function with respect to the metric $g$. Since $K_{g} \equiv 0$ and $\log r_{o}$ is harmonic and positive on $B_{x}(1)$ for all $x$ with $d_{g}(o, x) \geq 2 R$, where $B_{x}(1)$ is the geodesic ball of radius 1 centered at $x$ with respect to the metric $g$, the gradient estimate of [5] implies that there is a absolute constant $C_{1}$ such that

$$
\left|\nabla_{g} \log \left(r_{0}(x)\right)\right| \leq C_{1} \log \left(r_{0}(x)\right),
$$

for all $x$ with $d_{g}(o, x) \geq 2 R$. Hence, there is a constant $C_{2}>0$ such that

$$
v(x) \geq-C_{2} \log \left(r_{0}(x)\right)
$$

for all $x$ with $r_{0}(x)$ large enough. So $v+C_{2} \log \left(r_{0}(x)\right)$ is nonnegative harmonic near infinity in $\mathbb{R}^{2}$. Therefore, by the argument in Corollary 5.5 in [13],

$$
v(x)=u(x)+C_{3} \log \left(r_{0}(x)\right)
$$

near infinity for some bounded harmonic function $u$ and some constant $C_{3}$. In any case, $v$ is either bounded from above or bounded from below, so $v$ is a constant. Then putting back into the expression of $K_{g}$ and using $K_{g} \equiv 0$, we conclude that $|\phi|$ and hence $\phi$ is a nonzero constant.

Using Lemmas 1.2 and 1.3, we generalize the results of [22] to the following:

Proposition 1.4. If $\phi d z^{2} \in Q D(\mathbb{D})$ and $h(z)$ is a function in $C_{\text {loc }}^{\alpha}(\mathbb{D})$ satisfying $0<a^{2} \leq h(z) \leq b^{2}$ for all $z \in \mathbb{D}$. Then there exists a unique solution $w \in C_{\text {loc }}^{2, \alpha}(\mathbb{D})$ of

$$
\triangle_{p} w=h(z)\left(e^{2 w}-\|\phi\|^{2} e^{-2 w}\right)-1,
$$

such that $e^{2 w} d s_{p}^{2}$ defines a complete metric on $\mathbb{D}$ and that $\|\phi\| e^{-2 w} \leq 1$. 
Proof. Note that if $v$ is a solution given by Lemma 1.3, then $\tilde{v}=v-\log \rho$ satisfies

$$
\triangle_{p} \tilde{v}=c^{2}\left(e^{2 \tilde{v}}-\|\phi\|^{2} e^{-2 \tilde{v}}\right)-1 .
$$

By Lemma 1.3, there exist solutions $w_{a}$ and $w_{b}$ of

$$
\triangle_{p} w_{a}=a^{2}\left(e^{2 w_{a}}-\|\phi\|^{2} e^{-2 w_{a}}\right)-1,
$$

and

$$
\triangle_{p} w_{b}=b^{2}\left(e^{2 w_{b}}-\|\phi\|^{2} e^{-2 w_{b}}\right)-1,
$$

respectively such that $e^{2 w_{a}} \mathrm{~d} s_{p}^{2}$ and $e^{2 w_{b}} \mathrm{~d} s_{p}^{2}$ are complete metrics on $\mathbb{D}$ and satisfy $\|\phi\| e^{-2 w_{b}} \leq 1$ and $\|\phi\| e^{-2 w_{a}} \leq 1$. By Lemma 1.2 , we have $w_{b} \leq w_{a}$. Since $b^{2} \geq h(z) \geq a^{2}>0$ in $\mathbb{D},\|\phi\| e^{-2 w_{b}} \leq 1$, and $\|\phi\| e^{-2 w_{a}} \leq 1, w_{a}$ is a subsolution and $w_{b}$ is a supersolution of (1.1). Using the method of sub- and supersolutions as in [22] (with modification of the regularity of solutions), we conclude that there exists a solution $w \in C_{\mathrm{loc}}^{2, \alpha}(\mathbb{D})$ of (1.1) satisfying $w_{b} \leq$ $w \leq w_{a}$. Since $e^{2 w_{b}} \mathrm{~d} s_{p}^{2}$ is complete and satisfies $\|\phi\| e^{-2 w_{b}} \leq 1, e^{2 w} \mathrm{~d} s_{p}^{2}$ is also complete on $\mathbb{D}$ and $w$ satisfies $\|\phi\| e^{-2 w} \leq 1$. Finally, the uniqueness follows from Lemma 1.2. This completes the proof of the proposition.

Using Lemma 1.2 and the proof of the above proposition, we can obtain certain a-priori estimates on the solution $w$ given by Proposition 1.4 under suitable assumptions on $\phi \mathrm{d} z^{2}$ and the function $h$.

Proposition 1.5. Suppose $w \in C_{\text {loc }}^{2, \alpha}(\mathbb{D})$ satisfies

$$
\triangle_{p} w=h(z)\left(e^{2 w}-\|\phi\|^{2} e^{-2 w}\right)-1,
$$

for some $\phi(z) d z^{2} \in Q D(\mathbb{D})$ and for some function $h \in C_{\text {loc }}^{\alpha}(\mathbb{D})$ with $0 \leq a^{2} \leq$ $h(z) \leq b^{2}$, for all $z \in \mathbb{D}, e^{2 w} d s_{p}^{2}$ is complete on $\mathbb{D}$, and $\|\phi\| e^{-2 w} \leq 1$. Then

$$
w \geq-\log b .
$$

If, in addition, $\phi d z^{2} \in B Q D(\mathbb{D})$ and $a^{2}>0$, then we also have

$$
w \leq \frac{1}{2} \log \left(\frac{1+\sqrt{1+4 a^{4}\|\phi\|_{Q D}^{2}}}{2 a^{2}}\right) .
$$


Proof. Let $w_{b}$ as in the proof of Proposition 1.4. By Lemma 1.2 with $w_{1}=w_{b}$, $w_{2}=w, h_{1} \equiv b^{2}>0$ and $h_{2}=h \leq b^{2}$, we conclude that $w_{b} \leq w$. By Lemma $1.3, w_{b} \geq-\log b$, and hence $w \geq-\log b$. If in addition $a^{2}>0$ and $\|\phi\|_{Q D}<\infty$, then we let $w_{a}$ be the function as in the proof of Proposition 1.4, and use Lemma 1.2 as before to conclude that $w \leq w_{a}$. By Lemma 1.3, we have

$$
\begin{aligned}
w & \leq w_{a} \\
& \leq \frac{1}{2} \log \left(\frac{1+\sqrt{1+4 a^{4}\|\phi\|_{Q D}^{2}}}{2 a^{2}}\right)
\end{aligned}
$$

and the proof of the proposition is completed.

If $\|\phi\|_{Q D}=\infty$, then we do not have the uniform upper bound for $w$. However, if $a>0$, we still have a local upper bound as follows.

Proposition 1.6. Let $\phi d z^{2} \in Q D(\mathbb{D})$, and let $w$ be as in Proposition 1.5 with some $h \in C_{\text {loc }}^{\alpha}(\mathbb{D})$, and $0<a^{2} \leq h(z) \leq b^{2}$ in $\mathbb{D}$. Then for any $z \in \mathbb{D}$ and $r$ such that $0<r<1-|z|$,

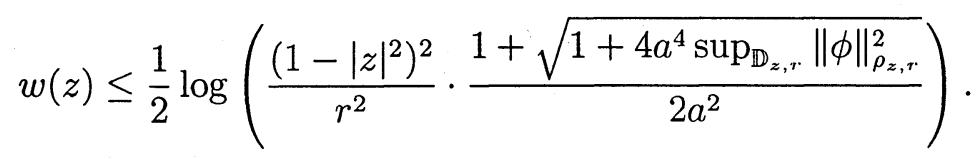

Proof. By Lemma 1.3, there is a unique solution $v$ of

$$
\left\{\begin{array}{l}
\triangle_{p_{z, r}} v=a^{2}\left(e^{2 v}-\|\phi\|_{\rho_{z, r}}^{2} e^{-2 v}\right)-1 \\
e^{2 v} \mathrm{~d} s_{\rho_{z, r}}^{2} \quad \text { complete on } \mathbb{D}_{z, r} \\
\|\phi\|_{\rho_{z, r}} e^{-2 v} \leq 1
\end{array}\right.
$$

where $\triangle_{p_{z, r}}$ is the Laplacian on $\mathbb{D}_{z, r}$ with respect to the Poincaré metric. Note that $\sup _{\mathbb{D}_{z, r}}\|\phi\|_{\rho_{z, r}} \leq r^{2} \sup _{\mathbb{D}_{z, r}}|\phi| / 4<\infty$. We have, as in the proof of Proposition 1.5,

$$
v \leq \frac{1}{2} \log \left(\frac{1+\sqrt{1+4 a^{4} \sup _{\mathbb{D}_{z, r}}\|\phi\|_{\rho_{z, r}}^{2}}}{2 a^{2}}\right) .
$$

Let $\tilde{w}=w+\log \rho-\log \rho_{z, r}$. Then by a straight forward calculation we have

$$
\triangle_{p_{z, r}} \tilde{w}=h(\zeta)\left(e^{2 \tilde{w}}-\|\phi\|_{\rho_{z, r}}^{2} e^{-2 \tilde{w}}\right)-1
$$


and $\|\phi\|_{\rho_{z, r}} e^{-2 \tilde{w}} \leq 1$ on $\mathbb{D}_{z, r}$. By Lemma 1.2 with obvious modifications, we get $\tilde{w} \leq v$ and hence

$$
w(\zeta) \leq \log \frac{\rho_{z, r}(\zeta)}{\rho(\zeta)}+\frac{1}{2} \log \left(\frac{1+\sqrt{1+4 a^{4} \sup _{\mathbb{D}_{z, r}}\|\phi\|_{\rho_{z, r}}^{2}}}{2 a^{2}}\right)
$$

Putting $\zeta=z$, we get

$$
w(z) \leq \log \left(\frac{1-|z|^{2}}{r}\right)+\frac{1}{2} \log \left(\frac{1+\sqrt{1+4 a^{4} \sup _{\mathbb{D}_{z, r}}\|\phi\|_{\rho_{z, r}}^{2}}}{2 a^{2}}\right),
$$

which is the desired inequality.

Proposition 1.7. Let $\phi d z^{2} \in Q D(\mathbb{D})$, and let $w$ be as in Proposition 1.5 with some $h \in C_{\text {loc }}^{\alpha}(\mathbb{D})$, and $0<a^{2} \leq h(z) \leq b^{2}$. in $\mathbb{D}$. Then for any $r$ and $r^{\prime}, 0<r<r^{\prime}<1$, there exists $C_{3}>0$, which depends only on $r, r^{\prime}, a, b$, $\sup _{\mathbb{D}_{r^{\prime}}}|\phi|$, and $\|h\|_{C^{\alpha}\left(\mathbb{D}_{r^{\prime}}\right)}$ such that

$$
\|w\|_{C^{2, \alpha}\left(\mathbb{D}_{r}\right)} \leq C_{3}
$$

Proof. By Propositions 1.5 and 1.6, $w$ and $\triangle_{0} w$ are bounded on $\mathbb{D}_{r_{1}}=\mathbb{D}_{\left(r+r^{\prime}\right) / 2}$ by constants depending only on $a, b$, and $\sup _{\mathbb{D}_{r^{\prime}}}|\phi|$, where $\triangle_{0}$ denotes the Euclidean Laplacian. So there exists a constant $C_{4}>0$, which depends only on the bounds of $w$ and $\triangle_{0} w$ on $\mathbb{D}_{r_{1}}$ (see Chapter 4 in [10]), such that

$$
\sup _{\mathbb{D}_{r_{2}}}|\nabla w| \leq C_{4}
$$

where $r_{2}=\frac{1}{2}\left(r+r_{1}\right)$. Since $w$ satisfies equation (1.1), the $C^{0, \alpha}$ norm of $\triangle_{0} w$ on $\mathbb{D}_{r_{2}}$ is bounded by a constant depending only on $r, r^{\prime}$, the bound of $w$ and the bound on $\Delta_{0} w$ in $\mathbb{D}_{r_{1}}$. By the interior Schauder estimates (see Chapter 4 in [10], for example), there is a constant $C_{3}>0$, depending only on $r, r^{\prime}, C^{0}$ bound of $w$ and $C^{0, \alpha}$ bound of $\triangle_{0} w$ on $\mathbb{D}_{r_{2}}$, such that

$$
\|w\|_{C^{2, \alpha}\left(\mathbb{D}_{r}\right)} \leq C_{3}
$$

Since $\left|\Delta_{0} w\right|$ is bounded on $\mathbb{D}_{r_{1}}$ by a constant depending only on $a, b$ and $\sup _{\mathbb{D}_{r^{\prime}}}|\phi|$, the proposition follows.

As a consequence of this proposition, we have the following: 
Proposition 1.8. Let $h_{n} \in C_{\text {loc }}^{\alpha}(\mathbb{D})$. Suppose that $h_{n} \rightarrow h_{0}$ in $C_{\text {loc }}^{\alpha}(\mathbb{D})$ as $n \rightarrow \infty$, and that $0<a^{2} \leq h_{n}(z) \leq b^{2}$ for $n=0,1,2, \ldots$ Let $w_{n}$ be solutions of

$$
\triangle_{p} w_{n}=h_{n}(z)\left(e^{2 w_{n}}-\|\phi\|^{2} e^{-2 w_{n}}\right)-1
$$

such that $e^{2 w_{n}} d s_{p}^{2}$ complete and $\|\phi\| e^{-2 w_{n}} \leq 1$ for all $n=0,1,2, \cdots$. Then $w_{n}$ converges to $w_{0}$ in $C_{\text {loc }}^{2}(\mathbb{D})$ as $n \rightarrow \infty$.

Proof. Since $h_{n}$ converges to $h_{0}$ in $C_{\mathrm{loc}}^{\alpha}(\mathbb{D})$, for each $r^{\prime}<1$, there is a uniform bound of $\left\|h_{n}\right\|_{C^{\alpha}\left(\mathbb{D}_{r^{\prime}}\right)}, n=1,2, \cdots$. By Proposition 1.7 , for any $r<r^{\prime}$, there exists a constant $C_{5}>0$, which depends only on $r, r^{\prime}, a, b, \sup _{\mathbb{D}_{r^{\prime}}}|\phi|$, and the uniform bound of $\left\|h_{n}\right\|_{C^{\alpha}\left(\mathbb{D}_{r^{\prime}}\right)}$ such that $\left\|w_{n}\right\|_{C^{2, \alpha}\left(B_{r}\right)} \leq C_{5}$. Therefore, any subsequence of $\left\{w_{n}\right\}$ has a subsequence converges $C^{2}$ locally uniformly on compact subsets. Let $f \in C^{2}(\mathbb{D})$ be the limiting function of a subsequence of $\left\{w_{n}\right\}$ in $C_{\text {loc }}^{2}(\mathbb{D})$. Then $f$ satisfies

$$
\triangle_{p} f=h_{0}(z)\left(e^{2 f}-\|\phi\|^{2} e^{-2 f}\right)-1
$$

since $h_{n}$ converges to $h_{0}$. By Proposition 1.5, we have $w_{n}(z) \geq-\log b$ on $\mathbb{D}$ for all $n=1,2, \cdots$. Hence $f \geq-\log b$, and $e^{2 f} \mathrm{~d} s_{p}^{2}$ is a complete metric on $\mathbb{D}$. By assumption, $\|\phi\| e^{-2 w_{n}} \leq 1$ for all $n$, so $f$ also satisfies $\|\phi\| e^{-2 f} \leq 1$. The uniqueness part of Proposition 1.4 then implies that $f=w_{0}$. Since $f$ is an arbitrary limiting function, $\left\{w_{n}\right\}$ converges to $w_{0}$ in $C_{\mathrm{loc}}^{2}(\mathbb{D})$. This completes the proof of the proposition.

Finally, let us prove a global "dilatation" estimate which is useful in later sections.

Proposition 1.9. Let $\phi d z^{2} \in B D Q(\mathbb{D})$, and let $w$ be a solution of equation (1.1) for some $h \in C_{\text {loc }}^{\alpha}(\mathbb{D})$ so that $0 \leq h(z) \leq b^{2}$ in $\mathbb{D}$ for some constant $b>0$, such that $e^{-2 w}\|\phi\| \leq 1$ and $e^{2 w} d s_{p}^{2}$ is complete. Then there exists a positive constant $k<1$, which depends only on $b^{2}\|\phi\|_{Q D}$, such that

$$
\sup _{z \in \mathbb{D}}\|\phi\| e^{-2 w} \leq k
$$

Proof. Let $v$ be the solution in Lemma 1.3 with $c=b$ and $\tilde{v}=v-\log \rho$. By the generalized maximum principle Lemma 1.1 and the fact that $h \leq b^{2}$, we 
have $w \geq \tilde{v}$. Therefore by Lemma 1.3, there is a constant $k<1$ depending only on $b^{2}\|\phi\|_{Q D}$ such that

$$
e^{-2 w}\|\phi\| \leq e^{-2 \tilde{v}}\|\phi\|=e^{-2 v}|\phi| \leq k
$$

\section{BASIC EXISTEnCE TheOREM}

Let $N$ be a complete simply connected surface with Gaussian curvature $K_{N}$ satisfying $-b^{2} \leq K_{N} \leq-a^{2}<0$ for some $b \geq a>0$. It is well known that $N$ is conformally equivalent to the hyperbolic 2 -space. Hence, $N$ can be represented as $N=\left(\mathbb{D}, \mathrm{d} s^{2}\right)$ with $\mathrm{d} s^{2}=\sigma^{2} \mathrm{~d} s_{p}^{2}$ for some function $\sigma$. It is also well-known that the first eigenvalue for the Laplacian for functions of $N$ is positive. Therefore, $N$ is a hyperbolic $\mathrm{CH}$ surface. Note that by [17], the conformal factor $\sigma$ is bounded. In this section, we generalize the result in $[22]$ to this class of surfaces, and show that given any $\phi \mathrm{d} z^{2} \in B D Q(\mathbb{D})$, there is a quasi-conformal harmonic diffeomorphism $u$ from $\mathbb{D}$ onto $N$, such that the Hopf differential of $u$ is $\phi \mathrm{d} z^{2}$. In [22], $N$ is the hyperbolic space and the harmonic diffeomorphism is constructed using the theory of constant mean curvature cut in Minkowski space. Here, we will use a more direct method. Observe that if $u$ is a harmonic diffeomorphism, and let $e^{2 w}=\|\partial u\|^{2}$, then $u$ and $w$ satisfy the system:

$$
\left\{\begin{array}{l}
\triangle_{p} w=-K_{\sigma}(u(z))\left(e^{2 w}-\|\phi\|^{2} e^{-2 w}\right)-1, \\
u_{\bar{z}}=\rho^{-2} \bar{\phi} e^{-2 w} u_{z} .
\end{array}\right.
$$

This system is coupled, unless $K_{N}$ is constant. Our first main step is to study the system and prove the following:

Theorem 2.1. Given any holomorphic quadratic differential $\phi d z^{2} \in B Q D(\mathbb{D})$, and any complete smooth conformal metric $\sigma^{2}(z) d s_{p}^{2}$ on $\mathbb{D}$ with curvature $K_{\sigma}$ satisfying $-b^{2} \leq K_{\sigma} \leq-a^{2}<0$, there exist a smooth function $w$ and a quasiconformal diffeomorphism $u$ from $\mathbb{D}$ onto $\mathbb{D}$, such that

(1) $w$ and $u$ satisfy the system of equations:

$$
\left\{\begin{array}{l}
\triangle_{p} w=-K_{\sigma}(u)\left(e^{2 w}-\|\phi\|^{2} e^{-2 w}\right)-1, \\
u_{\bar{z}}=\rho^{-2} \bar{\phi} e^{-2 w} u_{z}
\end{array}\right.
$$

(2) $u$ is normalized so that $u(0)=0$ and $u(1)=1$, 
(3) $e^{2 w} d s_{p}^{2}$ is complete,

(4) $\|\phi\| e^{-2 w} \leq k<1$,

where $k$ is the constant given by Proposition 1.9 which depends only on $b^{2}\|\phi\|_{Q D}$.

Remark 2.1. The quasi-conformal diffeomorphism $u$ may be normalized by other methods. For example, we may normalize $u$ so that $u$ fixed three given points on the boundary of $\mathbb{D}$. The problem on the uniqueness of the solutions $w$ and $u$ will be discussed in section 4 .

We will use the Schauder fixed point theorem to prove Theorem 2.1. Let

$$
\mathfrak{B}=\left\{w \in C^{0}(\mathbb{D}):\|w\|_{\mathfrak{B}}<+\infty\right\}
$$

where

$$
\|w\|_{\mathfrak{B}}=\sup _{\mathbb{D}} \rho^{-2}(z)|w(z)|
$$

where $\rho^{2}(z)=\frac{4}{\left(1-|z|^{2}\right)^{2}}$. Here we may take any other continuous function $f>0$ in stead of $\rho$ with the property that $1 / f(z) \rightarrow 0$ as $|z| \rightarrow 1$. Note that if $w \in C^{0}(\mathbb{D})$ and $\sup _{\mathbb{D}}|w|<\infty$, then $w \in \mathfrak{B}$. It is easy to see that $\left(\mathfrak{B},\|\cdot\|_{\mathfrak{B}}\right)$ is a Banach space. It is also easy to see that $w_{n} \rightarrow w$ in $\mathfrak{B}$ implies that $w_{n} \rightarrow w$ uniformly on compact sets on $\mathbb{D}$ as continuous functions. Let

$$
\mathfrak{S}=\left\{w \in \mathfrak{B}: \sup _{\mathbb{D}}|w| \leq C_{1} \text { and } \sup _{\mathbb{D}}\left(\|\phi\| e^{-2 w}\right) \leq k\right\}
$$

where

$$
C_{1}=\max \left(\log b, \frac{1}{2} \log \left(\frac{1+\sqrt{1+4 a^{4}\|\phi\|_{Q D}^{2}}}{2 a^{2}}\right)\right)
$$

and $0<k<1$ is the constant given by Proposition 1.9 which depends only on $b^{2}\|\phi\|_{Q D}$. Since $b \geq a>0, C_{1} \geq 0$. In fact, $C_{1}>0$ unless $b=a=1$ and $\|\phi\|_{Q D}=0$. It is also easy to see that $\mathfrak{S}$ is closed and convex in $\mathfrak{B}$. Let $w=v-\log \rho$, where $v$ is the solution given by Lemma 1.3 with $c=a$. By Lemma 1.3 and Proposition 1.9, $w \in \mathfrak{S}$. So $\mathfrak{S}$ is nonempty.

Now, given any holomorphic quadratic differential $\phi \mathrm{d} z^{2} \in B Q D(\mathbb{D})$, and any complete smooth conformal metric $\sigma^{2}(z) \mathrm{d} s_{p}^{2}$ on $\mathbb{D}$ with curvature $K_{\sigma}$ satisfying $-b^{2} \leq K_{\sigma} \leq-a^{2}<0$, we define a mapping $T$ from $\mathfrak{S}$ to $\mathfrak{B}$ as follows.

For any $w \in \mathfrak{S}$, since $\sup _{\mathbb{D}}\left(\|\phi\| e^{-2 w}\right) \leq k<1$, by Theorem A.1, Theorem A.2 and Theorem A.4, there is a unique quasi-conformal diffeomorphism $u$ 
from $\mathbb{D}$ onto $\mathbb{D}$ such that $u(0)=0$ and $u(1)=1$, and $u$ satisfies the Beltrami equation

$$
\frac{\partial u}{\partial \bar{z}}=\rho^{-2} \bar{\phi} e^{-2 w} \frac{\partial u}{\partial z}
$$

Furthermore, $u$ is in $C^{\alpha}(\mathbb{D})$ for some $\alpha>0$ depending only on $k$. By Proposition 1.4 , there exists a unique $v \in C^{2, \alpha}(\mathbb{D})$ such that

$$
\triangle_{p} v=-K_{\sigma}(u)\left(e^{2 v}-\|\phi\|^{2} e^{-2 v}\right)-1
$$

and $e^{2 v} \mathrm{~d} s_{p}^{2}$ defines a complete metric on $\mathbb{D}$ with $\|\phi\| e^{-2 v} \leq 1$. We define $T(w)$ to be the unique solution $v$. Propositions 1.5 and 1.9 imply that

$$
T \mathfrak{S} \subset \mathfrak{S}
$$

Lemma 2.2. The map $T$ is continuous on $\mathfrak{S}$.

Proof. Let $w_{n} \rightarrow w$ in $\mathfrak{B}$, then $w_{n}$ converges locally uniformly to $w$ on compact subsets of $\mathbb{D}$. Let $u_{n}$ be the quasi-conformal diffeomorphism on $\mathbb{D}$ such that $u_{n}(0)=0, u_{n}(1)=1$, and

$$
\frac{\partial u_{n}}{\partial \bar{z}}=\rho^{-2} \bar{\phi} e^{-2 w_{n}} \frac{\partial u_{n}}{\partial z}
$$

Similarly, let $u$ be the quasi-conformal diffeomorphism on $\mathbb{D}$, such that $u(0)=$ $0, u(1)=1$, and

$$
\frac{\partial u}{\partial \bar{z}}=\rho^{-2} \bar{\phi} e^{-2 w} \frac{\partial u}{\partial z}
$$

First of all, Proposition A.5 implies that $u_{n}$ converges locally uniformly to $u$ in $\mathbb{D}$. So, for any $0<r<1$, there is a $0<r^{\prime}<1$ such that $u_{n}\left(\mathbb{D}_{r}\right) \subset \mathbb{D}_{r^{\prime}}$ for all $n$. Hence we conclude that the $C^{\alpha}\left(\mathbb{D}_{r}\right)$ norm of $K_{\sigma}\left(u_{n}\right)$ have a uniform bound which depends only on $r, a, b,\|\phi\|_{Q D}$, and $\left\|K_{\sigma}\right\|_{C^{1}\left(\mathbb{D}_{r^{\prime}}\right)}$. By Proposition 1.8 , we conclude that $T\left(w_{n}\right) \rightarrow T(w)$ locally uniformly in $\mathbb{D}$ as $n \rightarrow \infty$. Since $\sup _{\mathbb{D}}\left|T\left(w_{n}\right)\right| \leq C_{1}, \sup _{\mathbb{D}}|T(w)| \leq C_{1}$, and $\rho^{-1}(z) \rightarrow 0$ as $|z| \rightarrow 1$, it is easy to see that $T\left(w_{n}\right)$ also converges to $T(w)$ in the norm $\|\cdot\|_{\mathfrak{B}}$. This completes the proof of the continuity of $T$.

Lemma 2.3. The image $T \mathfrak{S}$ of $\mathfrak{S}$ under the map $T$ is precompact in $\mathfrak{B}$. 
Proof: By Proposition 1.7, and the proof of Lemma 2.2, any sequence $\left\{T w_{n}\right\}$ in $T \mathfrak{S}$ has a subsequence which converges locally uniformly on compact subsets of $\mathbb{D}$. Since $\sup _{\mathbb{D}}\left|T w_{n}\right| \leq C_{1}$ for all $n$, and $\rho^{-1}(z) \rightarrow 0$ as $|z| \rightarrow 1$, the subsequence is also convergent with respect to the norm $\|\cdot\|_{\mathfrak{B}}$. Therefore $T$ is precompact.

Proof of Theorem 2.1. By Lemmas 2.2 and 2.3 and the Schauder fixed points theorem (see for example [10]), $T$ has a fixed point $w \in \mathfrak{S}$. By the definition of $T$, we have a quasi-conformal diffeomorphism $u$ on $\mathbb{D}$, such that $u(0)=0$, $u(1)=1$,

$$
\frac{\partial u}{\partial \bar{z}}=\rho^{-2} \bar{\phi} e^{-2 w} \frac{\partial u}{\partial z}
$$

and

$$
\Delta_{p} w=-K_{\sigma}(u)\left(e^{2 w}-\|\phi\|^{2} e^{-2 w}\right)-1,
$$

such that $w \in C_{l o c}^{2, \alpha}(\mathbb{D}), e^{2 w} \mathrm{~d} s_{p}^{2}$ is complete, and $\|\phi\| e^{-2 w} \leq k$ on $\mathbb{D}$. By Theorem A.4 and using a boot-strap argument, one shows that $w$ is in fact smooth. This completes the proof of the theorem.

Next, we will prove that the quasi-conformal map $u$ obtained in Theorem 2.1 is in fact a harmonic diffeomorphism.

Lemma 2.4. Let $N=\left(\mathbb{D}, \sigma^{2}|d u|^{2}\right)$ be a complete surface. Suppose the Gaussian curvature of $N$ satisfies $-b^{2} \leq K_{N} \leq-a^{2}<0$ for some constant $b \geq a>0$. Let $\phi d z^{2} \in B Q D(\mathbb{D})$. Suppose $w$ is a real valued smooth function on $\mathbb{D}$ and $u$ is a quasi-conformal diffeomorphism from $\mathbb{D}$ onto $\mathbb{D}$ such that

(i) $w$ satisfies the equation

$$
\Delta_{p} w=-K_{N}(u)\left(e^{2 w}-\|\phi\|^{2} e^{-2 w}\right)-1,
$$

(ii) $e^{2 w} d s_{p}^{2}$ is complete,

(iii) $e^{-2 w}\|\phi\| \leq k$ everywhere for some $0<k<1$,

(iv) $u$ satisfies the Beltrami equation

$$
\frac{\partial u}{\partial \bar{z}}=e^{-2 w} \rho^{-2} \bar{\phi} \frac{\partial u}{\partial z}
$$


Then $u$ is a harmonic diffeomorphism from $\left(\mathbb{D}, d s_{p}^{2}\right)$ onto $N$, such that the Hopf differential of $u$ is given by $\phi d z^{2}$ and $\|\partial u\|^{2}=e^{2 w}$.

Proof. The method of proof is to define a metric in the target so that $u$ is harmonic, and then we will show that the metric we defined is in fact the original metric on $N$. Since $u$ is a smooth quasi-conformal diffeomorphism, the Jacobian of $u$ is positive and $u_{z}=\partial u / \partial z \neq 0$ everywhere on $\mathbb{D}$. Let $\sigma_{1}^{2}(\zeta)|\mathrm{d} \zeta|^{2}$ be a conformal metric in the target. In order that $u$ is harmonic under this metric, it is necessary that the $\partial$-energy density of $u$ with respect to the new metric satisfies

$$
\|\partial u\|^{2}=e^{2 w}=\frac{\sigma_{1}^{2}(u(z))}{\rho^{2}(z)}\left|u_{z}(z)\right|^{2},
$$

where $\rho^{2}|d z|^{2}$ is the Poincaré metric on $\mathbb{D}$. Hence, we define

$$
\sigma_{1}^{2}(\zeta)=e^{2 w(z)} \rho^{2}(z) /\left|u_{z}(z)\right|^{2}
$$

where $z=u^{-1}(\zeta)$. It is easy to see that $\sigma_{1}^{2}(u)|\mathrm{d} u|^{2}$ is well defined since $u$ is a diffeomorphism from $\mathbb{D}$ onto $\mathbb{D}$. We claim that $\sigma_{1}^{2}(u)|d u|^{2}$ is complete. In fact, at any $z \in \mathbb{D}$,

$$
\begin{aligned}
u^{*}\left(\sigma_{1}^{2}(\zeta)|\mathrm{d} \zeta|^{2}\right) & =\sigma_{1}^{2}(u) u^{*}\left(|\mathrm{~d} \zeta|^{2}\right) \\
& =\sigma_{1}^{2}(u)\left|u_{z} \mathrm{~d} z+u_{\bar{z}} \mathrm{~d} \bar{z}\right|^{2} \\
& =\sigma_{1}^{2}(u)\left(u_{z} \mathrm{~d} z+u_{\bar{z}} \mathrm{~d} \bar{z}\right)\left(\bar{u}_{\bar{z}} \mathrm{~d} \bar{z}+\bar{u}_{z} \mathrm{~d} z\right) \\
& =\sigma_{1}^{2}(u)\left(u_{z} \mathrm{~d} z+e^{-2 w} \rho^{-2} \bar{\phi} u_{z} \mathrm{~d} \bar{z}\right)\left(\bar{u}_{\bar{z}} \mathrm{~d} \bar{z}+e^{-2 w} \rho^{-2} \phi \bar{u}_{\bar{z}} \mathrm{~d} z\right) \\
& =\sigma_{1}^{2}(u)\left|u_{z}\right|^{2}\left\{e^{-2 w} \rho^{-2}\left(\phi \mathrm{d} z^{2}+\bar{\phi} \mathrm{d} \bar{z}^{2}\right)+\left(1+e^{-4 w} \rho^{-4}|\phi|^{2}\right)|\mathrm{d} z|^{2}\right\} \\
& =\phi \mathrm{d} z^{2}+\bar{\phi} \mathrm{d} \bar{z}^{2}+\rho^{2}\left(e^{2 w}+\|\phi\|^{2} e^{-2 w}\right)|\mathrm{d} z|^{2},
\end{aligned}
$$

where we have used assumption (iv) and the definition of $\sigma_{1}$. Direct computations show that the two eigenvalues of the symmetric two tensor $\phi \mathrm{d} z^{2}+$ $\bar{\phi} \mathrm{d} \bar{z}^{2}+\rho^{2}\left(e^{2 w}+\|\phi\|^{2} e^{-2 w}\right)|\mathrm{d} z|^{2}$ are $\rho^{2}\left(e^{w}+\|\phi\| e^{-w}\right)^{2}$ and $\rho^{2}\left(e^{w}-\|\phi\| e^{-w}\right)^{2}$. By (2.1) and assumption (iii),

$$
u^{*}\left(\sigma_{1}^{2}|\mathrm{~d} \zeta|^{2}\right) \geq(1-k)^{2} e^{2 w} \rho^{2}|\mathrm{~d} z|^{2}
$$

Assumption (ii) implies that $u^{*}\left(\sigma_{1}^{2}|\mathrm{~d} \zeta|^{2}\right)$ is a complete metric and so is $\sigma_{1}^{2}|\mathrm{~d} \zeta|^{2}$. 
Next, we want to prove that $u$ is harmonic with respect to the metric $\sigma_{1}(u)|d u|^{2}$. Note that

$$
\begin{aligned}
\sigma_{1}^{2}(u) u_{z} \bar{u}_{z} & =e^{2 w} \rho^{2}\left|u_{z}\right|^{-2} u_{z} \bar{u}_{z} \\
& =e^{2 w} \rho^{2}\left|u_{z}\right|^{-2} u_{z} e^{-2 w} \rho^{-2} \phi \bar{u}_{\bar{z}} \\
& =\phi,
\end{aligned}
$$

where we have used again the assumption (iv). Since $\phi$ is holomorphic, we have

$$
\begin{aligned}
0 & =\phi_{\bar{z}} \\
& =\sigma_{1}^{2} u_{z \bar{z}} \bar{u}_{z}+2 \sigma_{1}\left(\sigma_{1}\right)_{u} u_{\bar{z}} u_{z} \bar{u}_{z}+\sigma_{1}^{2} u_{z} \bar{u}_{z \bar{z}}+2 \sigma_{1}\left(\sigma_{1}\right)_{\bar{u}} \bar{u}_{\bar{z}} u_{z} \bar{u}_{z} \\
& =\sigma_{1}^{2}\left(\bar{u}_{z} A+u_{z} \bar{A}\right)
\end{aligned}
$$

where $A=u_{z \bar{z}}+2\left(\log \sigma_{1}\right)_{u} u_{z} u_{\bar{z}}$. By assumptions (iii), (iv) and the fact that $u_{z} \neq 0$, we have $A=0$. Therefore $u$ is a harmonic diffeomorphism from $\left(\mathbb{D}, \mathrm{d} s_{p}^{2}\right)$ onto $N_{1}=\left(\mathbb{D}, \sigma_{1}^{2}|\mathrm{~d} u|^{2}\right)$ with Hopf differential given by $\phi \mathrm{d} z^{2}$ and $e^{2 w}$ is the $\partial$-energy density of $u$ with respect to $\sigma_{1}^{2}|\mathrm{~d} u|^{2}$.

Finally, we want to prove that $\sigma_{1}=\sigma$. Using the Bochner formula in [20], we see that $w$ also satisfies

$$
\Delta_{P} w=-K_{N_{1}}(u)\left(e^{2 w}-\|\phi\|^{2} e^{-2 w}\right)-1 .
$$

So we have $K_{N}(u(z))=K_{N_{1}}(u(z))$ for all $z \in \mathbb{D}$ by assumption (i) and (iii). Since $u$ is a diffeomorphism, $K_{N}(u)=K_{N_{1}}(u)$ for all $u \in \mathbb{D}$. Hence we have

$$
\begin{aligned}
\frac{1}{\sigma^{2}} \Delta_{0} \log \sigma & =\frac{1}{\sigma_{1}^{2}} \Delta_{0} \log \sigma_{1} \\
& =-K(u),
\end{aligned}
$$

where $K(u)=K_{N}(u)=K_{N_{1}}(u)$. Therefore

$$
\Delta_{N} \log \frac{\sigma_{1}}{\sigma}=-K\left(\frac{\sigma_{1}^{2}}{\sigma^{2}}-1\right) .
$$

Since $N$ is complete and $K_{N}=K$ is pinched between two negative constants, by the generalized maximum principle Lemma 1.1, we have $\sigma_{1} \leq \sigma$. Using the fact that $N_{1}$ is complete, we prove similarly that $\sigma \leq \sigma_{1}$. Therefore $\sigma \equiv \sigma_{1}$. So $u$ is a harmonic diffeomorphism from $\left(\mathbb{D}, d s_{p}^{2}\right)$ onto $N$ with Hopf differential given by $\phi \mathrm{d} z^{2}$ and $\|\partial u\|^{2}=e^{2 w}$. This completes the proof of the lemma. 
By Theorem 2.1 and Lemma 2.4, we have the following particular case of the Main Theorem.

Theorem 2.5. Let $N=\left(\mathbb{D}, \sigma^{2}|d u|^{2}\right)$ be a complete surface such that the Gaussian curvature of $N$ satisfies $-b^{2} \leq K_{N} \leq-a^{2}<0$. Let $\phi d z^{2} \in B D Q(\mathbb{D})$. Then there is a quasi-conformal harmonic diffeomorphism $u$ from $\left(\mathbb{D}, d s_{p}^{2}\right)$ onto $N$ with Hopf differential given by $\phi d z^{2}$ and which is normalized so that $u(0)=0$ and $u(1)=1$.

Remark 2.2. If $N$ is the hyperbolic space, then $u$ can be realized as the Gauss map of a complete constant mean curvature spacelike surface of hyperbolic type in Minkowski three space, see [22]. The complete surface is in fact isometric to $\left(\mathbb{D}, e^{2 w} \mathrm{~d} s_{p}^{2}\right)$.

\section{Proof of the MaIn Theorem}

Base on the Theorem 2.5, which is a special case, we will prove in this section the Main Theorem in its full generality. Let us first assume that the sectional curvature $K_{N}$ of the target $N$, a hyperbolic $\mathrm{CH}$ surface, satisfies $-b^{2} \leq K_{N} \leq-a^{2}<0$ for some constants $b \geq a>0$.

Theorem 3.1. Let $N=\left(\mathbb{D}, \sigma^{2}|d u|^{2}\right)$ be a complete surface with Gaussian curvature $K_{N}$ satisfying $-b^{2} \leq K_{N} \leq-a^{2}<0$ for some constants $b \geq a>0$. Then given any holomorphic quadratic differential $\Phi=\phi d z^{2}$ on $\mathbb{D}_{R_{0}}$ with $0<R_{0} \leq \infty$, there is a harmonic map $u$ from $\mathbb{D}_{R_{0}}$ to $N$ such that its Hopf differential is $\Phi$. Moreover, if $R_{0} \neq \infty$ or $\phi$ is not a constant, then $u$ can be chosen to be a harmonic diffeomorphism into $N$.

Proof. If $\Phi \equiv 0$, we can take $u(z)=z / R_{0}$ for $R_{0} \neq \infty$ and the constant map for $R_{0}=\infty$. So from now on, we assume $\Phi \not \equiv 0$.

By Theorem 2.5, for any $R_{0}>R>0$, there is an orientation preserving harmonic diffeomorphism $u_{R}$ from $\mathbb{D}_{R}$ onto $N$ such that the Hopf differential of $u_{R}$ is $\Phi$ restricted on $\mathbb{D}_{R}$ and $u_{R}(0)=0$. In particular, $\left(u_{R}\right)_{z} \neq 0$ everywhere. The metric on the domain considered in Theorem 2.5 is the Poincaré metric. As it is well known, the harmonicity depends only on the conformal structure, not the metric. So we can regard $u_{R}$ as a harmonic map from $\left(\mathbb{D}_{R},|\mathrm{~d} z|^{2}\right)$, i.e. 
with Euclidean metric, as well. But we should mention, on the contrary, that the energy densities depend on the metric on the domain. For example, the $\partial$-energy density of $u_{R}$ is $\left\|\partial u_{R}\right\|=\sigma\left(u_{R}\right)\left|u_{z}\right|$ if we use the Euclidean metric on the domain.

As before, let us write $w_{R}=\log \left\|\partial u_{R}\right\|$. Then the Bochner formula in [20] takes the form

$$
\Delta_{0} w_{R}=-K_{N}\left(u_{R}\right)\left(e^{2 w_{R}}-|\phi|^{2} e^{-2 w_{R}}\right),
$$

since the Euclidean metric is flat. From Theorem 2.5 that $u_{R}$ is a diffeomorphism, we have

$$
e^{2 w_{R}}>|\phi|
$$

and $e^{2 w_{R}}|\mathrm{~d} z|^{2}$ is a complete metric on $\mathbb{D}_{R}$. For any $0<r<R<R_{0}$, by Lemma 1.3 , there is a function $v_{a}$ satisfying

$$
\Delta_{0} v_{a}=a^{2}\left(e^{2 v_{a}}-|\phi|^{2} e^{-2 v_{a}}\right)
$$

and $e^{-2 v_{a}}|\phi|<1$ on $\mathbb{D}_{r}$ such that $e^{2 v_{a}}|\mathrm{~d} z|^{2}$ is a complete metric on $\mathbb{D}_{r}$. By (3.1) and (3.2) we have

$$
b^{2}\left(e^{2 w_{R}}-|\phi|^{2} e^{-2 w_{R}}\right) \geq \Delta_{0} w_{R} \geq a^{2}\left(e^{2 w_{R}}-|\phi|^{2} e^{-2 w_{R}}\right) .
$$

Using Lemma 1.1, (3.3), the second inequality in (3.4), and the fact that $e^{2 v_{a}}|\mathrm{~d} z|^{2}$ is complete on $\mathbb{D}_{r}$, we see that

$$
w_{R} \leq v_{a}
$$

on $\mathbb{D}_{r}$. Hence for any fixed $r^{\prime}, 0<r^{\prime}<r<R_{0}$, there is a constant $C_{1}$ independent of $R$, such that for all $R_{0}>R>r, w_{R} \leq C_{1}$ on $\mathbb{D}_{r^{\prime}}$. By the definition of $w_{R}$ and the fact that $u_{R}$ is an orientation preserving diffeomorphism, we conclude that the energy density of $u_{R}$ are uniformly bounded on compact subsets of $\mathbb{D}_{R_{0}}$. Since $u_{R}(0)=0$ for all $R$, passing to a subsequence if necessary, $u_{R}$ converges uniformly in the $C^{\infty}$ sense to a harmonic map $u$ on compact subsets of $\mathbb{D}_{R_{0}}$. Moreover, it is easy to see that the Hopf differential of the limiting harmonic map $u$ is $\Phi$. So we have proved the first part of the theorem. 
To prove the second statement, we note that if $R_{0} \neq \infty$ or $\phi$ is not equal to a constant, then by Lemma 1.3 there is a function $v_{b}$ satisfying

$$
\Delta_{0} v_{b}=b^{2}\left(e^{2 v_{b}}-|\phi|^{2} e^{-2 v_{b}}\right)
$$

and $e^{2 v_{b}}>|\phi|$ on $\mathbb{D}_{R_{0}}$ such that $e^{2 v_{b}}|\mathrm{~d} z|^{2}$ is complete on $\mathbb{D}_{R_{0}}$. Using Lemma 1.1 , the first inequality in (3.4), (3.5), and the fact that $e^{2 w_{R}}|\mathrm{~d} z|^{2}$ is complete on $\mathbb{D}_{R}$, we see that

$$
w_{R} \geq v_{b}
$$

on $\mathbb{D}_{R}$. Since $e^{w_{R}}=\left\|\partial u_{R}\right\|=\sigma\left(u_{R}\right)\left|\left(u_{R}\right)_{z}\right|$ converges uniformly to $\|\partial u\|=$ $\sigma(u)\left|u_{z}\right|$ on compact sets in $\mathbb{D}_{R_{0}}$, we have $\|\partial u\|^{2} \geq e^{2 v_{b}}>|\phi|$ everywhere in $\mathbb{D}_{R_{0}}$. We conclude that the Jacobian $J(u)=\|\partial u\|^{2}-|\phi|^{2}\|\partial u\|^{-2}$ is positive everywhere in $\mathbb{D}_{R_{0}}$ and hence $u$ is a local diffeomorphism.

It remains to prove that $u$ is globally one-to-one. In fact, the inequalities $v_{b} \leq w_{R} \leq v_{a}$ and (3.2) not only imply the boundedness of $\|\partial u\|$ and the positivity of $J(u)$. They also imply that $J\left(u_{R}\right)$ is uniformly bounded away from zero on compact sets. Therefore, $u_{R}$ are local quasi-isometries with quasi-isometric constants independent of $R$. That is to say, for any point $p \in \mathbb{D}_{R_{0}}$ and $q=u(p)$, there is a neighborhood $U$ of $p$, a neighborhood $V$ of $q$ such that $u_{R}(U)$ is contained in $V, u_{R}$ maps $U$ onto $u_{R}(U)$ quasi-isometrically, and the quasi-isometric constants can be chosen independent of $R$ for $R<R_{0}$. Since $u_{R}$ is a diffeomorphism for each $R<R_{0}$, it is not hard to see that $u$ must be one-to-one. This completes the proof of the theorem.

Remark 3.1. By the proof of the theorem, it is easy to see that, if $\phi \not \equiv 0$ the harmonic map satisfies $\|\partial u\|>0$ and $e^{2 w}|\mathrm{~d} z|^{2}=\|\partial u\|^{2}|\mathrm{~d} z|^{2}$ is complete on $\mathbb{D}_{R_{0}}$. In fact, $w \geq v_{b}$, where $v_{b}$ is the solution in (3.5).

Now, we are going to remove the pinching condition on Theorem 3.1 and complete our proof of the Main Theorem. Recall that a hyperbolic $\mathrm{CH}$ surface is a complete simply connected surface $N$ with Gaussian curvature $K_{N}$ of $N$ satisfying $-b^{2} \leq K_{N} \leq 0$, and with positive first eigenvalue for the Laplacian for functions. We want to construct harmonic maps from $\mathbb{H}$ or $\mathbb{R}^{2}$ into a hyperbolic $\mathrm{CH}$ surface with prescribed Hopf differential. Since the $K_{N}$ is not bounded above by a negative constant, we cannot use Proposition 1.6. One 
obvious way to overcome the difficulty is to perturb the metric on $N$ so that the perturbed metric has negatively pinched Gaussian curvature. One can do this provided that the first eigenvalue of $N$ is positive. If this is the case, then by the results in [17], $N$ can be represented as $\left(\mathbb{D}, e^{2 \psi} \mathrm{d} s_{p}^{2}\right)$ with $|\psi|$ uniformly bounded, where $\mathrm{d} s_{p}^{2}$ is the Poincare metric. This fact will be used in the following proof of the Main Theorem to perturb the metric. Note that, since $N$ is simply connected, it is well-known that the first eigenvalue $\lambda_{1}(N)$ is positive if $K_{N} \leq-a^{2}<0$.

Theorem 3.2 (Main Theorem). Let $N=\left(\mathbb{D}, e^{2 \psi} d s_{p}^{2}\right)$ be a hyperbolic $C H$ surface with Gaussian curvature $K_{N}$ satisfying $-b^{2} \leq K_{N} \leq 0$ for some constant $b>0$ and $\lambda_{1}(N)>0$. Then given any holomorphic quadratic differential $\Phi=\phi d z^{2}$ on $\mathbb{D}_{R_{0}}, R_{0}=1$ or $\infty$, there is a harmonic map $u$ from $\mathbb{D}_{R_{0}}$ to $N$ with Hopf differential given by $\Phi$. Moreover, if $R_{0}=1$ or $\phi$ is not a constant, then $u$ can be chosen to be a harmonic diffeomorphism into $N$. Futhermore, if $R_{0}=1$ and $\Phi \in B D Q(\mathbb{D})$, then $u$ can be chosen to be a quasi-conformal harmonic diffeomorphism onto $N$.

Proof. If $\Phi=0$, then the identity mapping and constant mapping are the required harmonic diffeomorphism and harmonic map for $R_{0}=1$ and $R_{0}=\infty$ respectively. So we only need to consider the case that $\Phi \neq 0$.

As we mentioned above, $|\psi|$ is uniformly bounded. So for all $0 \leq t \leq 1$, $e^{2 t \psi} \mathrm{d} s_{p}^{2}$ is quasi-isometric to $\mathrm{d} s_{p}^{2}$ and hence complete on $\mathbb{D}$. In fact, $e^{-2 \sup |\psi|} \leq$ $e^{2 t \psi} \leq e^{2 \sup |\psi|}$. Denote $\left(\mathbb{D}, e^{2 t \psi} \mathrm{d} s_{p}^{2}\right)$ by $N_{t}$, and let $K_{t}$ be the Gaussian curvature of $N_{t}$. Then

$$
\begin{aligned}
K_{t} & =-\frac{\Delta_{0}(t \psi)+\log \rho}{e^{2 t \psi} \rho^{2}} \\
& =-\frac{t \Delta_{0} \psi+\Delta_{0} \log \rho}{e^{2 t \psi \rho^{2}}} \\
& =-(1-t) e^{-2 t \psi}+t e^{2(1-t) \psi} K_{N}
\end{aligned}
$$

where $\mathrm{d} s_{p}^{2}=\rho^{2}|\mathrm{~d} u|^{2}$, and we have used the equation $\rho^{-2} \Delta_{0} \psi+1+K_{N} e^{2 \psi}=0$. Therefore,

$$
-b^{2} t e^{2(1-t) \sup |\psi|}-(1-t) e^{2 t \sup |\psi|} \leq K_{t} \leq-(1-t) e^{-2 t \sup |\psi|},
$$


for all $0 \leq t \leq 1$. And hence there exists a constant $\beta>0$ independent of $t$ such that

$$
-\beta^{2} \leq K_{t} \leq-(1-t) e^{-2 t \sup |\psi|}
$$

for all $0 \leq t \leq 1$. Since $-(1-t) e^{-2 t \sup |\psi|}<0$ for $0 \leq t<1$, by Theorem 3.1 there is a harmonic map $u_{t}$ from $\mathbb{D}_{R_{0}}$ to $N_{t}$ with $u_{t}(0)=0$ such that their Hopf differential is $\Phi$. Furthermore, as it is stated in Remark 3.1, we also have $\left\|\partial u_{t}\right\|>0$ for all $0 \leq t<1, e^{2 w_{t}}|\mathrm{~d} z|^{2}=\left\|\partial u_{t}\right\|^{2}|\mathrm{~d} z|^{2}$ is complete on $\mathbb{D}_{R_{0}}$, and $w_{t} \geq v_{\beta}$, where $v_{\beta}$ is the solution of (3.5) with $b=\beta$.

Case 1: If $R_{0}=1$ or $\phi$ is not a constant.

In this case, by Lemma 1.3 , we have $e^{-2 v_{\beta}}|\phi|<1$. Since $w_{t} \geq v_{\beta}$ for all $0 \leq t<1$, for any fixed $r, 0<r<R_{0}$, there is a constant $0<k<1$ independent of $t$ such that

$$
e^{-2 w_{t}}|\phi|<1-k
$$

in $\mathbb{D}_{r}$ for all $0 \leq t<1$. Therefore

$$
\frac{\left|\left(u_{t}\right)_{\bar{z}}\right|}{\left|\left(u_{t}\right)_{z}\right|}<1-k,
$$

on $\mathbb{D}_{r}$. Now, from the proof of Theorem 3.1, $u_{t}$ is a harmonic diffeomorphism into $N_{t}$. It is easy to see that $\lambda_{1}\left(N_{t}\right) \geq C>0$ for some constant independent of $t$. So a local version of Theorem 1.1 in [17] implies that the energy density of $u_{t}$ is locally uniformly bounded. Using $u_{t}(0)=0$ and the fact that $e^{2 t \psi} \rightarrow e^{2 \psi}$ as $t \rightarrow 1$, we conclude that there is a harmonic map $u$ from $\mathbb{D}_{R_{0}}$ into $N$ such that the Hopf differential of $u$ is $\Phi$. As in the proof of Theorem 3.1, one shows that $u$ is in fact a harmonic diffeomorphism into $N$.

Case 2: $R_{0}=\infty$ and $\phi$ is a nonzero constant.

In this case, $v_{\beta}=\frac{1}{2} \log |\phi|=c$ is a constant on $\mathbb{R}^{2}$. For all $0 \leq t<1$,

$$
\Delta_{0} w_{t}=-K_{t}\left(u_{t}\right)\left(e^{2 w_{t}}-|\phi|^{2} e^{-2 w_{t}}\right) \text {. }
$$

is also satisfied trivially by $c$. Note that $e^{2 w_{\iota}}|\mathrm{d} z|^{2}$ and $c|\mathrm{~d} z|^{2}$ are both complete on $\mathbb{R}^{2}$. Since $K_{t}$ is bounded between two negative constants, by Lemma 1.2 , $w_{t}=c$. Therefore the energy densities of $u_{t}$ are again uniformly bounded and we can get the required harmonic map from $\mathbb{D}_{\infty}=\mathbb{R}^{2}$ to $N$ as in case 1 . Note that we may not have a diffeomorphism in this case. 
Finally, for the last assertion of the theorem, we observe that, if $R_{0}=1$ and $\Phi \in B D Q(\mathbb{D})$, Theorem 2.5 and Proposition 1.9 imply that the harmonic maps $u_{t}$ given by Theorem 3.1 are quasi-conformal diffeomorphisms with dilatation bounded uniformly by a constant $0<k<1$ depending only on $\|\Phi\|_{Q D}$. Then Theorem A.3 asserts that the limiting harmonic map $u$, which is not a constant map, is also quasi-conformal. This completes the proof of the theorem.

\section{RESUltS ON UNIQUENESS}

In this section, we will prove some results on the uniqueness of harmonic diffeomorphisms with the same Hopf differential. The following theorem reduces this question of uniqueness to the question of uniqueness of the solutions of the scalar equation satisfied by the $\partial$-energy density of the harmonic diffeomorphism.

Theorem 4.1. Let $M=\left(\mathbb{D}_{r}, \rho^{2}|d z|^{2}\right)$, where $r=1$ or $r=\infty$ and $\rho^{2}|d z|^{2}$ is the Poincaré metric for $r=1$, and the Euclidean metric for $r=\infty$. Let $N$ be a simply connected surface with nonpositive curvature. Let $u_{1}$ and $u_{2}$ be two orientation preserving harmonic diffeomorphisms from $M$ into $N$ with the same Hopf differential and with images $\Omega_{1}$ and $\Omega_{2}$ respectively. Suppose that $\left\|\partial u_{1}\right\| \equiv\left\|\partial u_{2}\right\|$. Then $u_{2} \circ\left(u_{1}\right)^{-1}: \Omega_{1} \rightarrow \Omega_{2}$ is an isometry. If in addition, the metric of $N$ is analytic, then $u_{2}=\iota_{N} \circ u_{1}$ for some isometry $\iota_{N}$ of $N$.

Proof. Let $\sigma^{2}|d u|^{2}$ be the metric of $N$, where $u$ is a complex coordinate. Let $u_{i}, i=1,2$ are orientation preserving harmonic diffeomorphisms with the same Hopf differential $\phi \mathrm{d} z^{2}$. Note that $\left\|\partial u_{i}\right\|>0, i=1,2$. For any $z \in \mathbb{D}_{r}$, as in (2.1), the pull-back of the metric tensor on $N$ under $u_{i}$ is given by

$$
\left(u_{i}\right)^{*}\left(\sigma^{2}|\mathrm{~d} u|^{2}\right)=\phi \mathrm{d} z^{2}+\bar{\phi} \mathrm{d} \bar{z}^{2}+\rho^{2}\left(e^{2 w_{i}}+\|\phi\|^{2} e^{-2 w_{i}}\right)|\mathrm{d} z|^{2} .
$$

for $i=1,2$, where $w_{i}=\log \left\|\partial u_{i}\right\|$. By assumption, $w_{1}=w_{2}$, and hence

$$
\left(u_{1}\right)^{*}\left(\sigma^{2}|\mathrm{~d} u|^{2}\right)=\left(u_{2}\right)^{*}\left(\sigma^{2}|\mathrm{~d} u|^{2}\right) .
$$

From this, it is easy to see that $u_{2} \circ\left(u_{1}\right)^{-1}$ is an isometry from $\Omega_{1}$ onto $\Omega_{2}$. Since $N$ is simply connected with nonpositive curvature, $u_{2} \circ\left(u_{1}\right)^{-1}$ can be extended to a map from $N$ into $N$ which maps geodesics from some fixed point in $\Omega_{1}$ onto geodesics from its image in $\Omega_{2}$ under $u_{2} \circ\left(u_{1}\right)^{-1}$. If furthermore, 
the metric on $N$ is analytic, then the extended map must be an isometry of $N$. This completes the proof of the theorem.

Next, we want to study the problem on uniqueness of the solutions of the scalar equation satisfied by the $\partial$-energy density of a harmonic diffeomorphism. We need the following proposition, which may have interest in its own right.

Proposition 4.2. Let $\mathbb{H}=\left(\mathbb{D}, d s_{p}^{2}\right)$ be the Poincaré disk, and let $N$ be a hyperbolic $C H$ surface. Let $u$ be an orientation harmonic diffeomorphism from $\mathbb{H}$ into $N$ so that $e^{2 w} d s_{p}^{2}$ is complete, where $e^{2 w}=\|\partial u\|^{2}$. Suppose the Hopf differential $\phi d z^{2}$ of $u$ is in $B Q D(\mathbb{D})$. Then $u$ is surjective, quasi-conformal, and the energy density of $u$ is bounded.

Proof. By the definition of a hyperbolic $\mathrm{CH}$ surface, the Gaussian curvature $K_{N}$ of $N$ satisfies $-b^{2} \leq K_{N} \leq 0$ for some $b>0$. Since $u$ is a diffeomorphism, $e^{-2 w}\|\phi\|<1$. By the Bochner formula and Proposition 1.9, there is a constant $0<k<1$, such that

$$
\sup _{z \in \mathbb{D}}\|\phi\| e^{-2 w} \leq k
$$

Since $e^{2 w} \mathrm{~d} s_{p}^{2}$ is complete, by the computations right after (2.1) in the proof of Lemma 2.4, we see that the pull back of the metric of $N$ under $u$ must be complete. Here we have used (4.1), and the fact that $k<1$. Hence $u$ must be onto. By (4.1) again, $u$ is quasi-conformal. By [17], we conclude that $u$ has bounded energy density.

If $N$ is a hyperbolic $\mathrm{CH}$ surface, then the Gaussian curvature may not be bounded above away from 0 . So we cannot apply Lemma 2.1 directly. However, in some cases, the results in Lemma 2.1 are still true:

Lemma 4.3. Let $w_{1}$ and $w_{2}$ be two solutions of the equation

$$
\Delta_{p} w=h\left(e^{2 w}-\|\phi\|^{2} e^{-2 w}\right)-1
$$

where $\Delta_{p}$ is the Laplacian of $\mathbb{H}, \phi d z^{2} \in Q D(\mathbb{D})$, and $h$ is a continuous function on $\mathbb{H}$ with $0 \leq h \leq b^{2}$ for some constant $b>0$. Suppose $e^{2 w_{i}} d s_{p}^{2}$ is complete on $\mathbb{D}$, and $e^{-2 w_{i}}\|\phi\| \leq 1$, for $i=1,2$, so that $w_{1}-w_{2}$ is bounded. Then $w_{1}=w_{2}$. 
Proof. By the proof of Theorem 2.1 in [17], see also [22], one can prove that

$$
e^{2 w_{i}} \geq \frac{1}{b^{2}}
$$

for $i=1,2$. In fact, since $0 \leq h \leq b^{2}$, and $e^{2 w_{i}}\|\phi\| \leq 1$,

$$
e^{-2 w_{i}} \Delta_{p}\left(-w_{i}\right) \geq-b^{2}+e^{-2 w_{i}} \text {. }
$$

Using the fact that $e^{2 w_{i}} d s_{p}^{2}$ is complete, with curvature bounded below by $-b^{2}$, we see that $-w_{i}$ is bounded from above. By the generalized maximum principle Lemma 1.1, (4.2) follows. Let $\eta=w_{1}-w_{2}$. By assumptions, $\eta$ is bounded. Let $\alpha>1$, then by (4.2)

$$
\alpha w_{1}-w_{2} \geq(\alpha-1) \log \frac{1}{b}+\eta
$$

In particular, $\alpha w_{1}-w_{2}$ is bounded from below. By the generalized maximum principle Lemma 1.1, there is a sequence of point $z_{k}$ in $\mathbb{D}$, such that

$$
\inf _{\mathbb{D}}\left(\alpha w_{1}-w_{2}\right)=\lim _{k \rightarrow \infty}\left(\alpha w_{1}-w_{2}\right)\left(z_{k}\right)
$$

and

$$
\lim _{k \rightarrow \infty} \Delta\left(\alpha w_{1}-w_{2}\right)\left(z_{k}\right) \geq 0 .
$$

Since $\eta=w_{1}-w_{2}$ is bounded and $e^{-2 w_{2}}\|\phi\| \leq 1$, we may assume that

$$
\lim _{k \rightarrow \infty} \eta\left(z_{k}\right)=a
$$

and

$$
\lim _{k \rightarrow \infty} e^{-2 w_{2}}\|\phi\|\left(z_{k}\right)=m
$$

for some constant $a$ and $m$ with $0 \leq m \leq 1$. By (4.4) and the fact that $w_{i}$ satisfies the equation in the lemma, given $\epsilon>0$, we have

$$
-\epsilon \leq h\left(z_{k}\right)\left(\alpha e^{2 w_{1}\left(z_{k}\right)}-e^{2 w_{2}\left(z_{k}\right)}-\|\phi\|^{2}\left(z_{k}\right)\left(\alpha e^{-2 w_{1}\left(z_{k}\right)}-e^{-2 w_{2}\left(z_{k}\right)}\right)\right)-\alpha+1,
$$

if $k$ is large enough. Since $\alpha>1$, if we choose $\epsilon$ small enough, then

$$
0<h\left(z_{k}\right)\left(\alpha e^{\left.2 w_{(} z_{k}\right)}-e^{2 w_{2}\left(z_{k}\right)}-\|\phi\|^{2}\left(z_{k}\right)\left(\alpha e^{-2 w_{1}\left(z_{k}\right)}-e^{-2 w_{2}\left(z_{k}\right)}\right)\right),
$$

provided that $k$ is large enough. Since $h \geq 0$, we have

$$
0<\alpha e^{2 w_{1}\left(z_{k}\right)}-e^{2 w_{2}\left(z_{k}\right)}-\|\phi\|^{2}\left(z_{k}\right)\left(\alpha e^{-2 w_{1}\left(z_{k}\right)}-e^{-2 w_{2}\left(z_{k}\right)}\right)
$$


for $k$ large. Dividing the above inequality by $e^{2 w_{2}}$ and let $k \rightarrow \infty$, we have

$$
0 \leq \alpha e^{2 a}-1-m^{2}\left(\alpha e^{-2 a}-1\right)
$$

Let $\alpha_{i}>1$ so that $\alpha_{i} \rightarrow 1$. Denote the corresponding $a$ and $m$ by $a_{i}$ and $m_{i}$ respectively. For all $i$

$$
0 \leq \alpha_{i} e^{2 a_{i}}-1-\left(m_{i}\right)^{2}\left(\alpha_{i} e^{-2 a_{i}}-1\right)
$$

Since $a_{i}$ and $m_{i}$ are bounded, we may assume that $a_{i} \rightarrow \tilde{a}$ and $m_{i} \rightarrow \tilde{m}$. By the definition of the $a_{i}$ and $b_{i}$, and by (4.3), it is easy to see that

$$
\inf _{\mathbb{D}}\left(w_{1}-w_{2}\right) \geq \tilde{a}
$$

By (4.5), if we let $i \rightarrow \infty$, we get

$$
0 \leq e^{2 \tilde{a}}-1-\tilde{m}^{2}\left(e^{-2 \tilde{a}}-1\right) .
$$

It is then easy to see that $\tilde{a} \geq 0$. $\mathrm{By}(4.6)$, we have $w_{1} \geq w_{2}$. Similarly, one can prove that $w_{2} \geq w_{1}$. So $w_{1}=w_{2}$.

We will also need a similar result on $\mathbb{C}$. The proof is different.

Lemma 4.4. Let $w_{1}$ and $w_{2}$ be two solutions of the equation

$$
\Delta_{0} w=h\left(e^{2 w}-|\phi|^{2} e^{-2 w}\right)
$$

on $\mathbb{C}$, where $\Delta_{0}$ is the Euclidean Laplacian of $\mathbb{C}, \phi d z^{2} \in Q D(\mathbb{C})$, and $h$ is a continuous function on $\mathbb{C}$ with $h \geq 0$. Suppose that $w_{1}-w_{2}$ is bounded. Then $w_{1}-w_{2}$ is a constant. If in addition, $h$ is not identically 0 , then $w_{1}=w_{2}$.

Proof. Let $\eta=w_{1}-w_{2}$. Then

$$
\Delta_{0} \eta=h e^{2 w_{2}}\left(e^{2 \eta}-1-e^{-4 w_{2}}|\phi|^{2}\left(e^{-2 \eta}-1\right)\right) .
$$

It is easy to see that at $\eta>0, \Delta_{0} \eta \geq 0$, and at $\eta<0, \Delta_{0} \eta \leq 0$. Therefore, $\min \{\eta, 0\}$ is superharmonic and $\max \{\eta, 0\}$ is subharmonic. Since they are both bounded, and every bounded superharmonic or subharmonic function on $\mathbb{C}$ is constant, $\eta$ must be a constant. If in addition, $h$ is not identically 0 , then it is easy to see that the constant must be 0 . This completes the proof of the lemma. 
Remark 4.1. In general, we cannot expect $w_{i}$ to be bounded in the above two lemmas.

Theorem 4.5. Let $\mathbb{H}=\left(\mathbb{D}, d s_{p}^{2}\right)$ be the Poincaré disk and let $N$ be a hyperbolic $C H$ surface with Gaussian curvature $K_{N}$. Let $\phi d z^{2}$ be a holomorphic quadratic differential in $B Q D(\mathbb{D})$. Let $u_{1}$ and $u_{2}$ be two orientation preserving harmonic diffeomorphisms from $\mathbb{H}$ into $N$ with the same Hopf differential $\phi d z^{2}$. Suppose $e^{2 w_{i}} d s_{p}^{2}$ is complete on $\mathbb{D}$, for $i=1,2$, where $w_{i}=\log \left\|\partial u_{i}\right\|$, and suppose $K_{N}\left(u_{1}(z)\right)=K_{N}\left(u_{2}(z)\right)$ for all $z \in \mathbb{D}$. Then there is an isometry $\iota_{N}$ of $N$ such that $u_{2}=\iota_{N} \circ u_{1}$.

Proof. By Proposition 4.2, $u_{1}$ and $u_{2}$ are both surjective with bounded energy density. As in the proof of Lemma 4.3

$$
e^{2 w_{i}} \geq \frac{1}{b^{2}}
$$

for $i=1,2$, where $-b^{2}$ is the lower bound of $K_{N}$. Hence $w_{i}=\log \left\|\partial u_{i}\right\|$ is uniformly bounded in $\mathbb{H}$, for $i=1,2$. Since $u_{i}$ is a harmonic diffeomorphism, we have $e^{2 w_{i}}|\phi|<1$. Since we assume $K_{N}\left(u_{1}\right)=K_{N}\left(u_{2}\right)$, by the Bochner formula, both $w_{1}$ and $w_{2}$ satisfy the same equation:

$$
\Delta_{p} w=h\left(e^{2 w}-\|\phi\|^{2} e^{-2 w}\right)-1,
$$

where $h=-K_{N}\left(u_{1}\right)=-K_{N}\left(u_{2}\right), 0 \leq h \leq b^{2}$. By Lemma 4.3, we conclude that $w_{1}=w_{2}$. By Theorem 4.1, noting that $u_{1}$ and $u_{2}$ are both surjective, the theorem follows.

If the norm of $\phi d z^{2}$ is not uniformly bounded, or if the domain is $\mathbb{C}$, then the result in the previous theorem is still true if we make stronger assumptions that the curvature of $N$ is pinched between two negative constants and the metric is analytic.

Proposition 4.6. Let $M=\left(\mathbb{D}_{r}, \rho^{2}|d z|^{2}\right)$ for $r=1$ or $r=\infty$, where $\rho^{2}|d z|^{2}$ is the Poincaré metric if $r=1$, and $\rho^{2} \equiv 1$ if $r=\infty$. Let $N$ be a simply connected complete surface with Gaussian curvature $K_{N}$ pinched between two negative constants $-a^{2}$ and $-b^{2}$. Let $\phi d z^{2}$ be a holomorphic quadratic differential in $\mathbb{D}_{r}$, and let $u_{1}$ and $u_{2}$ be two orientation preserving harmonic diffeomorphisms from $M$ into $N$ with the same Hopf differential $\phi d z^{2}$. Suppose $e^{2 w_{i}} \rho^{2}|d z|^{2}$ is 
complete on $\mathbb{D}_{r}$, for $i=1,2$, where $w_{i}=\log \left\|\partial u_{i}\right\|$, and suppose $K_{N}\left(u_{1}(z)\right)=$ $K_{N}\left(u_{2}(z)\right)$ for all $z \in \mathbb{D}_{r}$. Then $u_{2} \circ\left(u_{1}\right)^{-1}$ is an isometry from $u_{1}(M)$ onto $u_{2}(M)$. If in addition the metric of $N$ is analytic, then there is an isometry $\iota_{N}$ of $N$ such that $u_{2}=\iota_{N} \circ u_{1}$.

Proof. The proof is similar to the proof of Theorem 4.1, by using Lemma 1.2, and Theorem 4.1. We omit the details.

If we assume $w_{1}-w_{2}$ is bounded, then we can remove the restriction that the curvature of $N$ is bounded above by a negative constant.

Proposition 4.7. Let $M=\left(\mathbb{D}_{r}, \rho^{2}|d z|^{2}\right)$ for $r=1$ or $r=\infty$, where $\rho^{2}|d z|^{2}$ is the Poincaré metric if $r=1$, and $\rho^{2} \equiv 1$ if $r=\infty$. Let $N$ be a simply connected complete surface with Gaussian curvature $K_{N}$ bounded between $-b^{2}$ and 0 . Let $\phi d z^{2}$ be a holomorphic quadratic differential in $\mathbb{D}_{r}$ and let $u_{1}$ and $u_{2}$ be two orientation preserving harmonic diffeomorphisms from $M$ into $N$ with the same Hopf differential $\phi d z^{2}$, where $\phi$ is not a constant. Suppose $e^{2 w_{i}} \rho^{2}|d z|^{2}$ is complete on $\mathbb{D}_{r}$, for $i=1,2$, where $w_{i}=\log \left\|\partial u_{i}\right\|$, and suppose $K_{N}\left(u_{1}(z)\right)=K_{N}\left(u_{2}(z)\right)$ for all $z \in \mathbb{D}_{r}$. If $w_{1}-w_{2}$ is bounded, then $u_{2} \circ\left(u_{1}\right)^{-1}$ is an isometry from $u_{1}(M)$ onto $u_{2}(M)$. If in addition the metric of $N$ is analytic, then there is an isometry $\iota_{N}$ of $N$ such that $u_{2}=\iota_{N} \circ u_{1}$.

Proof. In case $r=1$, the proof is similar to the proof of the previous proposition, except we use Lemma 4.3 rather than Lemma 2.1. If $r=\infty$, then we can use Lemma 4.4 to get the result, provided we can prove $K_{N}\left(u_{1}\right)=K_{N}\left(u_{2}\right)$ is not identically 0 . To prove this, note that if $K_{N}\left(u_{1}\right)$ is identically 0 , then $w_{1}$ is harmonic by the Bochner formula. By the proof of Lemma 4.3, $w_{1}$ is bounded from below. So it must be a constant. However, $u_{1}$ is a diffeomorphism implies that $|\phi| \leq e^{2 w_{1}}$. Hence $\phi$ is bounded. By the Liouville theorem for holomorphic function, $\phi$ must be constant. This contradicts the assumption on $\phi$. The proof of the proposition is completed.

Remark 4.2. If $N$ is the hyperbolic two space, then $K_{N}$ is identically -1 , and the condition $K_{N}\left(u_{1}\right)=K_{N}\left(u_{2}\right)$ is automatically satisfied. In this case, the metric is obviously analytic. Hence Theorem 4.5 and Proposition 4.6 are 
generalizations of the results in [22] and [4]. Here, the results have been proved without using the properties of constant mean curvature cuts.

\section{Appendix A.}

In this appendix, we collect some facts about quasiconformal mappings which are used in this paper. The main reference is Letho-Virtanen's book [12] and Ahlfors' book [1]. The first result we need is the following:

Theorem A.1. Let $\Omega$ and $\Omega^{\prime}$ be simply-connected domains which are conformally equivalent. Suppose $\mu \in L_{\infty}(\Omega)$ satisfies $\|\mu\|_{\infty}<1$. Then there exists a quasiconformal homeomorphism $u: \Omega \rightarrow \Omega^{\prime}$ such that

$$
\frac{\partial u}{\partial \bar{z}}=\mu \frac{\partial u}{\partial z} \quad \text { almost everywhere. }
$$

The quasiconformal homeomorphism $u$ is unique up to a conformal transformation on $\Omega^{\prime}$.

The next result gives the uniform Hölder estimate for normalized family of quasiconformal mappings.

Theorem A.2. Let $u$ be a quasiconformal homeomorphism from $\mathbb{D}$ onto $\mathbb{D}$, such that the dilatation of $u$ satisfies $|\mu|=\left|u_{\bar{z}} / u_{z}\right| \leq k<1$ almost everywhere, for some $k<1$. Suppose $u(0)=0$ or u fixes three points on the boundary, $u$ is Hölder with exponent $(1-k) /(1+k)$ on $\mathbb{D}$ and the Hölder norm over $\mathbb{D}$ is bounded by a constant depenending only on $k$ and the method of normalization.

Proof. See Chapter 3 in [1] or Chapter 2 of [12].

From this, we have:

Theorem A.3. Let $\left\{u_{n}\right\}$ be a sequence of quasiconformal homeomorphism from $\mathbb{D}$ onto $\mathbb{D}$ with uniform dilatation bound, i.e. there is a constant $k<$ 1 such that $\left|\mu_{n}\right|=\left|\left(u_{n}\right)_{\bar{z}} /\left(u_{n}\right)_{z}\right| \leq k<1$. Suppose one of the following conditions holds.

(1) $\left\{u_{n}\right\}$ fixes a point in the interior of $\mathbb{D}$; or

(2) $\left\{u_{n}\right\}$ fixes three points on the boundary of $\mathbb{D}$.

Then $\left\{u_{n}\right\}$ is normal in $\mathbb{D}$, i.e. any subsequence in $\left\{u_{n}\right\}$ has a subsequence converges locally uniformly on compact subset in $\mathbb{D}$. 
For applying the Beltrami equation to study harmonic maps, we need also the following regularity result.

Theorem A.4. Suppose the complex dilatation of a quasiconformal homeomorphism $u$ from $\mathbb{D}$ onto $\mathbb{D}$ is in $C_{\text {loc }}^{\alpha}(\mathbb{D})$. Then $u$ is regular, i.e. $u$ is differentiable and its Jacobian never vanish on $\mathbb{D}$. Moreover, if the complex dilatation of $u$ is in $C_{l o c}^{k, \alpha}(\mathbb{D}), k \geq 0$, then $u$ is in $C_{l o c}^{k+1, \alpha}(\mathbb{D})$

Proof. This first statement is a special case of Theorem 7.1 in Chapter 5, Section 7.1 of [12]. For the second statement, one can result [18].

Finally let us prove a continuity result for normalized quasiconformal homeomorphism form $\mathbb{D}$ to $\mathbb{D}$.

Proposition A.5. Suppose the complex dilatation $\mu_{n}$ of $u_{n}: \mathbb{D} \rightarrow \mathbb{D}$ converges a.e. to $\mu_{0}$, the complex dilatation of $u_{0}: \mathbb{D} \rightarrow \mathbb{D}$, and $\left\{u_{n}\right\}$ and $u_{0}$ are normalized so that they fix the sume three points on the boundary or fix the same point in the interior and the same point on the boundary. Then $u_{n}$ converges locally uniformly to $u_{0}$.

Proof. First we notice that $\left\{u_{n}\right\}$ is a normal family. Hence if we can show that $u_{0}$ is the unique limiting function of the sequence, then $u_{n}$ will actually converges to $u_{0}$. Let $f$ be a limiting mapping from $\mathbb{D}$ to $\mathbb{D}$. Then by Chapter 2, section 5.6, Theorem 5.5 in [12], $f$ is either a quasiconformal homeomorphism from $\mathbb{D}$ onto $\mathbb{D}$ or mapping $\mathbb{D}$ to a boundary point of $\mathbb{D}$. Since $u_{n}$ are normalized, the later case cannot happen. Then Theorem 5.2 in Chapter 4, section 5.6 of [12] implies that $f$ and $u_{0}$ have the same complex dilatation $\mu_{0}$. Therefore the normalization condition implies that $f=u_{0}$. This completes the proof of the proposition.

\section{REFERENCES}

1. Ahlfors, L., Lectures on Quasiconformal Mappings, Van Nostrand, 1966.

2. Akutagawa, K., Harmonic diffeomorphisms of the hyperbolic plane, to appear in Trans. Amer. Math. Soc.

3. Akutagawa, K., Nishikawa, S. and Tachikawa, A., Harmonic maps between unbounded convex polyhedra in hyperbolic spaces, Invent. Math. 115 (1994) 391-404.

4. $\mathrm{Au}, \mathrm{T}$. and Wan, T., Parabolic constant mean curvature spacelike surfaces, to appear Proc. Amer. Math. Soc. 
5. Cheng, S. Y. and Yau, S.-T., Differential equations on Riemannian manifolds and their geometric applications, Comm. Pure Appl. Math. 28 (1975), 333-354.

6. Choi, H. I. and Treibergs, A., New examples of harmonic diffeomorphisms of the hyperbolic plane to itself, Manuscripta Math. 62 (1988), 249-256.

7. Choi, H. I. and Treibergs, A., Gauss map of spacelike constant mean curvature hypersurface of Minkowski space, J. Differential Geom. 32 (1990), 775-817.

8. Choi, H. I. and Treibergs, A., Constructing harmonic maps into the hyperbolic space, Proceedings of Symposia in Pure Mathematics, 54 (1993) Part I, 101-109.

9. Donnelly, H., Dirichlet problem at infinity for harmonic maps - rank one symmetric spaces, preprint.

10. Gilbarg, D. and Trudinger, N. S., Elliptic Partial Differential Equations of Second Order, 2nd ed., Springer-Verlag, New York 1983.

11. Jost, J., Two-dimensional Geometric Variational Problems, John Wiley \& Sons, 1991.

12. Lehto, O. and Virtanen, K. I., Quasiconformal Mappings in the Plane, SpringerVerlag, 1973.

13. Li, P. and Tam, L.-F., Positive harmonic functions on complete manifolds with non-negative curvature outside a compact set, Annal Math. 125 (1987), 171-207.

14. Li, P. and Tam, L.-F., The heat equation and harmonic maps of complete manifolds, Invent. Math. 105 (1991), 1-46.

15. Li, P. and Tam, L.-F., Uniqueness and regularity of proper harmonic maps, Ann. Math. 136 (1992), 169-203.

16. Li, P. and Tam, L.-F., Uniqueness and regularity of proper harmonic maps II, Indiana U. Math. J. 42 (1993), 593-635.

17. Li, P., Tam, L.-F. and Wang, J., Harmonic diffeomorphisms between hyperbolic Hamdamard manifolds, to appear Jour. Geom. Anal.

18. Spivak, M., A Comprehensive Introduction to Differential Geometry, Vol. 4, 2nd ed., Publish or Perish Inc., Berkeley 1979.

19. Schoen, R., The role of harmonic mappings in rigidity and deformation problems, preprint.

20. Schoen, R. and Yau, S.-T., On univalent harmonic maps between surfaces, Invent. Math. 44 (1978), 265-278.

21. Tam, L.-F. and Wan, Tom Y.-H., Quasi-conformal harmonic diffeomorphisms and the universal Tiechmüller space, to appear in J. Diff. Geom.

22. Wan, T., Constant mean curvature surface, harmonic maps, and universal Teichmüller space, J. Diff. Geom. 35 (1992), 643-657.

23. Wang, J.-P., The heat flow and harmonic maps between complete manifolds, thesis (1994), University of California, Irvine.

24. Wolf, M., The Teichmüller theory of harmonic maps, J. Diff. Geom. 29 (1989), 449-479.

University of California, Irvine, U. S. A.

E-mail address: ltam@math.uci.edu

The Chinese University of Hong Kong, Hong Kong

E-mail address: tomwan@cuhk.hk

ReCeived November 2, 1994. 